\title{
ATP6AP2 variant impairs CNS development and neuronal survival to cause fulminant neurodegeneration
}

Takuo Hirose, ${ }^{1}$ Alfredo Cabrera-Socorro, ${ }^{2,3,4}$ David Chitayat, ${ }^{5,6,7}$ Thomas Lemonnier, ${ }^{2,3,4}$ Olivier Féraud, ${ }^{8,9}$

Carmen Cifuentes-Diaz, ${ }^{2,3,4}$ Nicolas Gervasi, ${ }^{2,3,4}$ Cedric Mombereau, ${ }^{2,3,4}$ Tanay Ghosh, ${ }^{2,3,4}$ Loredana Stoica, ${ }^{2,3,4}$ Jeanne d'Arc Al Bacha, ${ }^{1,3,4,10,11}$ Hiroshi Yamada, ${ }^{12}$ Marcel A. Lauterbach, ${ }^{13}$ Marc Guillon, ${ }^{13}$ Kiriko Kaneko, ${ }^{14}$ Joy W. Norris, ${ }^{15}$ Komudi Siriwardena, ${ }^{6}$ Susan Blasér, ${ }^{6}$ Jérémie Teillon, ${ }^{1,16,17}$ Roberto Mendoza-Londono, ${ }^{6}$ Marion Russeau, ${ }^{2,3,4}$ Julien Hadoux, ${ }^{8,9}$ Sadayoshi Ito, ${ }^{18}$ Pierre Corvol, ${ }^{1,16,17}$ Maria G. Matheus, ${ }^{19}$ Kenton R. Holden, ${ }^{20}$ Kohji Takei, ${ }^{12}$ Valentina Emiliani, ${ }^{13}$ Annelise Bennaceur-Griscelli, ${ }^{8,9,21,22}$ Charles E. Schwartz, ${ }^{15}$ Genevieve Nguyen, ${ }^{1,16,17}$ and Matthias Groszer ${ }^{2,3,4}$

\begin{abstract}
'Collège de France, Center for Interdisciplinary Research in Biology, Paris, France. IINSERM, UMR-S 1270, Paris, France. ${ }^{3}$ Sorbonne Université, Paris, France. IInstitut du Fer à Moulin, Paris, France. ${ }^{5}$ Prenatal Diagnosis and Medical Genetics Program, Department of Obstetrics and Gynecology, Mount Sinai Hospital, University of Toronto, Toronto, Ontario, Canada. ${ }^{6}$ Division of Clinical and Metabolic Genetics and 'Department of Diagnostic Imaging, Division of Pediatric Neuroradiology, The Hospital for Sick Children, University of Toronto, Toronto, Ontario, Canada. ${ }^{8}$ INSERM, UMR 935, ESTeam Paris Sud, SFR André Lwoff, Université Paris Sud, Villejuif, France. ${ }^{9}$ Infrastructure Nationale INCESTEM, Université Paris Sud, INSERM, Paris, France. ${ }^{10}$ Laboratory of Applied Biotechnology, Azm Center for the Research in Biotechnology and Its Applications, Doctoral School for Sciences and Technology, Lebanese University, Tripoli, Lebanon. "Reviva Regenerative Medicine Center, Human Genetic Center, Middle East Institute of Health Hospital, Bsalim, Lebanon. ${ }^{12}$ Department of Neuroscience, Okayama University Graduate School of Medicine, Dentistry and Pharmaceutical Sciences, Okayama, Japan. ${ }^{13}$ WavefrontEngineering Microscopy Group, Neurophotonics Laboratory, CNRS, UMR 8250, Université Paris Descartes, Sorbonne Paris Cité, Paris, France. ${ }^{14}$ Department of Endocrinology and Applied Medical Science, Tohoku University Graduate School of Medicine, Sendai, Japan. ${ }^{15}$ Creenwood Genetic Center, Greenwood, South Carolina, USA. ${ }^{16}$ INSERM, U1050, Paris, France. ${ }^{17}$ CNRS, UMR 7241, Paris, France. ${ }^{18}$ Division of Nephrology, Endocrinology and Vascular Medicine, Department of Medicine, Tohoku University Graduate School of Medicine, Sendai, Japan. ${ }^{19}$ Department of Neurosciences (Neurology) and ${ }^{20}$ Department of

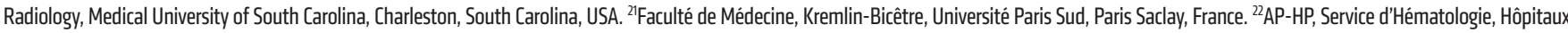
Universitaires Paris Sud, Hôpital Paul Brousse, Villejuif, France.
\end{abstract}

\begin{abstract}
Vacuolar $\mathrm{H}^{+}$-ATPase-dependent (V-ATPase-dependent) functions are critical for neural proteostasis and are involved in neurodegeneration and brain tumorigenesis. We identified a patient with fulminant neurodegeneration of the developing brain carrying a de novo splice site variant in ATP6AP2 encoding an accessory protein of the V-ATPase. Functional studies of induced pluripotent stem cell-derived (iPSC-derived) neurons from this patient revealed reduced spontaneous activity and severe deficiency in lysosomal acidification and protein degradation leading to neuronal cell death. These deficiencies could be rescued by expression of full-length ATP6AP2. Conditional deletion of Atp6ap2 in developing mouse brain impaired V-ATPase-dependent functions, causing impaired neural stem cell self-renewal, premature neuronal differentiation, and apoptosis resulting in degeneration of nearly the entire cortex. In vitro studies revealed that ATP6AP2 deficiency decreases V-ATPase membrane assembly and increases endosomal-lysosomal fusion. We conclude that ATP6AP2 is a key mediator of V-ATPase-dependent signaling and protein degradation in the developing human central nervous system.
\end{abstract}

\section{Introduction}

The vacuolar $\mathrm{H}^{+}$-adenosine triphosphatases (V-ATPases) are proton pumps present on endomembranes of all cells and acidify intracellular compartments, which is critical for numerous cellular processes, such as protein trafficking, maturation, recycling, or degradation (1). In addition, V-ATPases have also been involved in acidification-independent roles such as membrane fusion or secretion (2).

Authorship note: TH, ACS, DC, and TL contributed equally to this work. Conflict of interest: $\mathrm{CN}$ and $\mathrm{M}$. Groszer are inventors on the European patent PCT/ EP2013053538.

Copyright: @ 2019, American Society for Clinical Investigation.

Submitted: November 13, 2014; Accepted: March 5, 2019; Published: April 15, 2019.

Reference information: J Clin Invest. 2019;129(5):2145-2162.

https://doi.org/10.1172/JCl79990.
In lysosomes, V-ATPases regulate the optimal acidic $\mathrm{pH}$ for diverse enzymes to degrade macromolecules delivered from endocytic and autophagic pathways. In addition, lysosomal V-ATPases function as docking platform and amino acid sensors to regulate the activity of the mechanistic target of rapamycin complex 1 (mTORC1), a master regulator of cell growth and autophagy (3). Although disruptions of lysosomal V-ATPasemediated functions may impair protein homeostasis (proteostasis) in different tissues, they are frequently linked to neurodegenerative disorders (4).

ATP6AP2 (ATPase, $\mathrm{H}^{+}$transporting, lysosomal accessory protein 2) on Xp11.4 encodes a type I transmembrane protein mostly studied for its role as (pro)renin receptor in the reninangiotensin system (RAS) involved in blood pressure regulation (5). The ATP6AP2 protein is mainly composed of an extracellular 
A

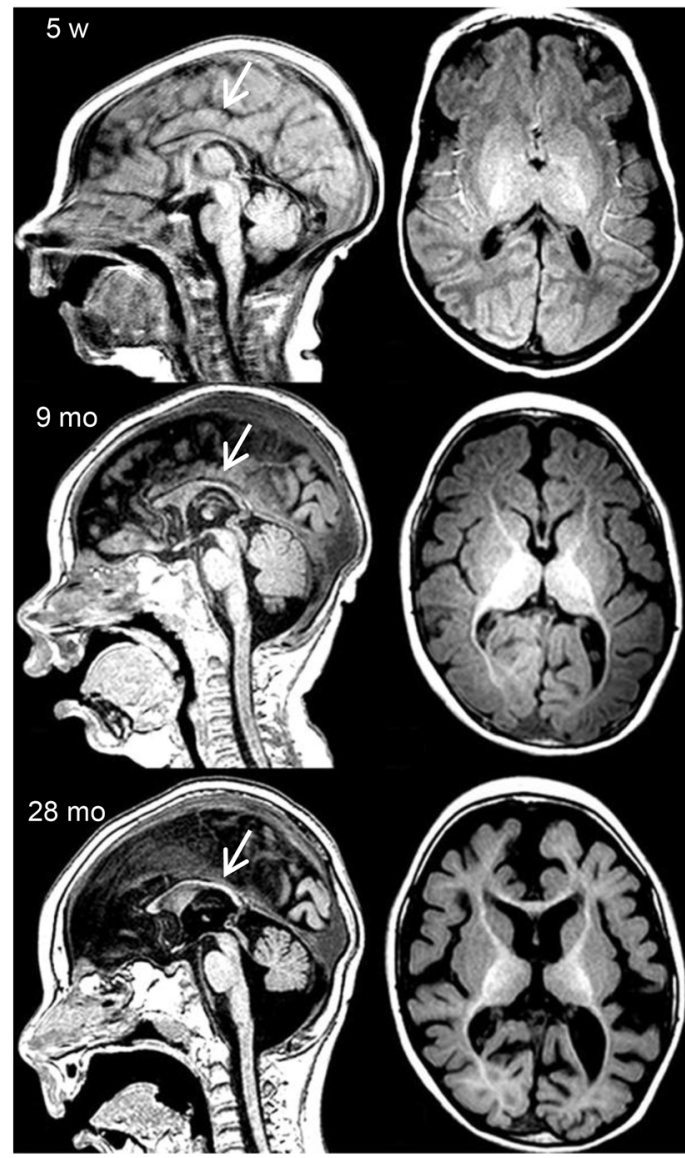

C

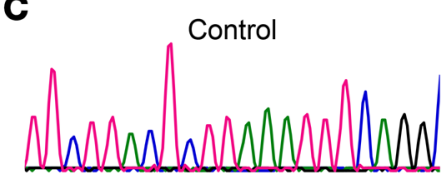

TTCTTACTCTTAAATTTCAGG

Patient

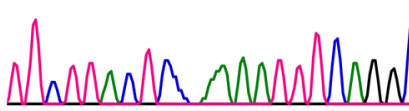

TTCTTACTC--AAATTTCAGG
D

\author{
Ex 3 Forward
}

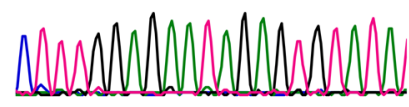

CTTTGGAGAATAGAgTGTATA

Ex 5 Reverse

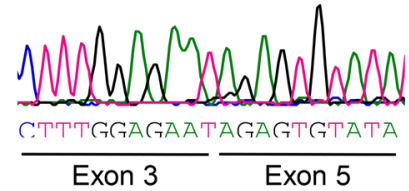

E

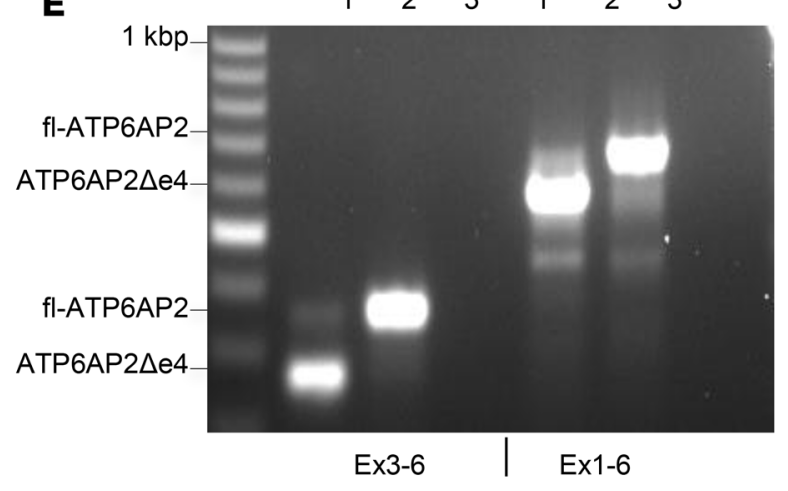

B

ATP6AP2
c.301-11_301-10delTT

ttgcgttctt actcttaaat ttcagGCAGT TCCTTTTAGT

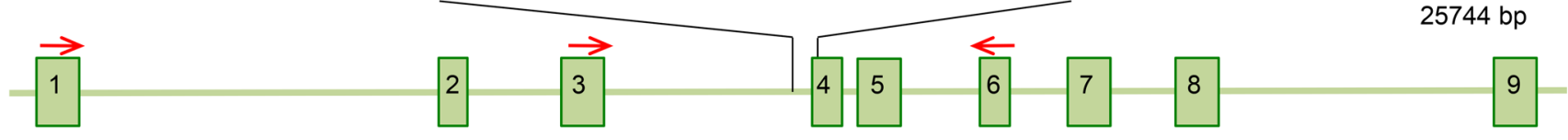

Figure 1. Fulminant neurodegeneration in patient carrying ATP6AP2 (c.301-11_301-10delTT) variant. (A) Sequential brain MRI of patient 1 carrying the ATP6AP2 (c.301-11_301-10delTT) variant at 5 weeks, 9 months, and 28 months of age. Sagittal (left) and axial (right) T1-weighted MR images at 5 weeks showed a thin, poorly developing corpus callosum (arrow) and diffuse volume loss of the brain parenchyma, enlarged ventricles, and prominent sulci. At 9 months the MRI revealed delayed myelination, a persistently thin hypoplastic corpus callosum (arrow), and parenchymal volume loss. At 28 months the MRI showed a markedly thin corpus callosum (arrow), minimal myelination throughout the brain, and significant parenchymal gray and white matter volume losses. (B) ATP6AP2 genomic organization and position of c.301-11_301-10delTT variant. Red arrows: primers used for RT-PCR. (C) Chromatogram of genomic DNA sequencing shows TT deletion in ATP6AP2 in the affected individual. (D) Chromatogram of cDNA sequencing shows exon 4 deletion. (E) RT-PCR analysis of patient fibroblast with $f$ I-ATP6AP2 and a shorter product representing approximately $80 \%$ of $A T P 6 A P 2$ transcripts. Lane 1, patient; lane 2, control; lane $3, \mathrm{H}_{2} \mathrm{O}$.

(pro)renin binding domain that can be secreted after cleavage by furin; a transmembrane domain; and a short cytoplasmic domain (6). The transmembrane/cytoplasmic part is present in species without renin, and the extracellular domain is only conserved in vertebrates. This suggests divergent functions of the ATP6AP2 domains and that its role in the RAS was acquired during vertebrate evolution (7). Accumulating evidence now indicates that ATP6AP2 has important functions apart from the RAS, as a V-ATPase accessory protein (8-10).

Recent studies in yeast and flies suggest that ATP6AP2 contributes to the assembly of the V-ATPase proton pore in the endoplasmic reticulum (11).
ATP6AP2 is highly expressed in the CNS, and human genetic studies suggested its potential involvement in diverse neuropsychiatric disorders. For instance, 2 synonymous variants in ATP6AP2 have been found to cosegregate in families with X-linked intellectual disability (XLID) and epilepsy (c.321C>T, p.D107D) (12) and $\mathrm{X}$-linked parkinsonism with spasticity (XPDS) (c.345C $>\mathrm{T}, \mathrm{p} . \mathrm{S} 115 \mathrm{~S}$ ) (13). Intriguingly, both variants associated with these diverse disorders increase skipping of exon 4 (ATP6AP2Ae4) that encodes part of the extracellular domain, resulting in ATP6AP2 $\triangle e 4$ in about $50 \%$ of ATP6AP2 transcripts. However, the causative role of ATP6AP2 has been questioned (14), since a potential splicing variant (NM_005765.2; c.859-2A $>$ C) resulting in a truncated pro- 


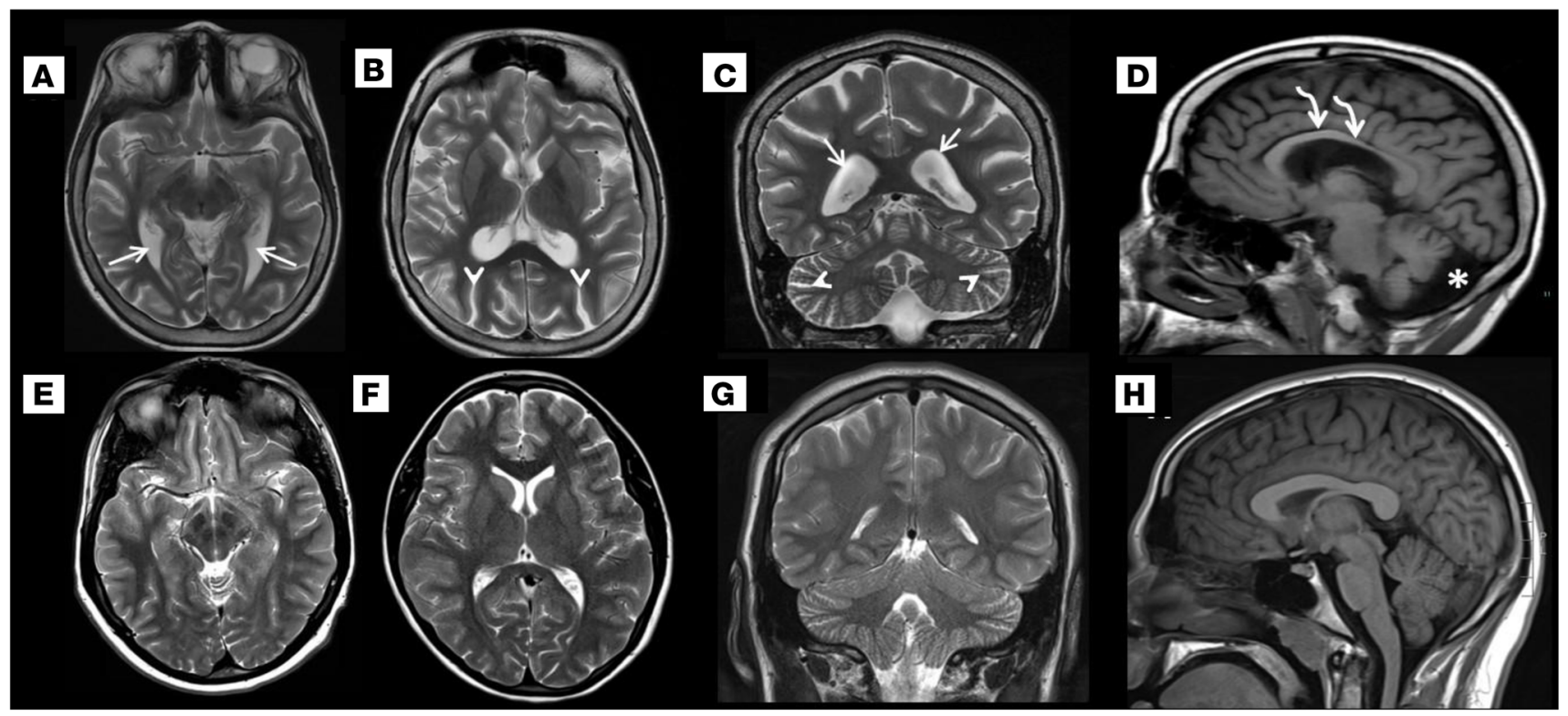

I $\quad A T P 6 A P 2$

c.321C>T (p.D107D)

$25744 \mathrm{bp}$

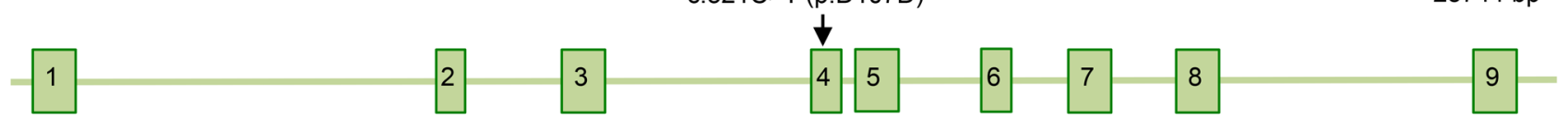

\begin{tabular}{|c|c|c|c|c|}
\hline J & fl-ATP6AP2 & Exon 4 & \multicolumn{2}{|l|}{ Furin cleavage site } \\
\hline 1 & 17 & 101 & $\checkmark 303$ & 23 \\
\hline SP & & & TM & Cyt \\
\hline
\end{tabular}

K $\quad$ ATP6AP2 $\triangle \mathrm{e} 4$

\begin{tabular}{|l|l|l|l|l|}
\hline \multicolumn{1}{|c|}{17} & & \multicolumn{2}{|c|}{318} \\
\hline SP & & & TM Cyt \\
\hline
\end{tabular}

Figure 2. Cerebral atrophy in a patient carrying the ATP6AP2 c.321C >T (p.D107D) variant. (A-H) Brain MRI of the 15-year-old male patient carrying the c.321C > T variant in exon 4 (OMIM 300423) and of an age-matched normal subject. Patient axial T2-weighted MR brain images (A and $\mathbf{B}$ ) and normal subject brain images (E and $\mathbf{F}$ ) for comparison. Patient coronal T2-weighted MR image (C) and normal subject (C). Patient MR images (A-C) exhibit diffuse parenchymal volume loss of gray and white matter. This is observed in the white matter tracts that travel adjacent to the prominently enlarged lateral ventricles (white arrows), as well as the prominent sulci (white arrowheads) in the cerebral hemispheres and cerebellum. Supratentorial and infratentorial parenchymal volume loss involves the cerebral hemispheres and cerebellum symmetrically. Sagittal T1-weighted MR brain image (D) compared with normal subject (H) demonstrates significantly reduced white matter volume of the corpus callosum (curved white arrows). There is also significant white matter volume loss in the patient cerebellum (D) with increased cerebrospinal fluid (asterisk) in the prominent posterior fossa mega cisterna magna. (I) ATP6AP2 genomic locus and positions of variant c.321C>T (p.D107D). (J and K) Schematics of ATP6AP2 and ATP6AP2 $\triangle \mathrm{e} 4$.

tein is present in the Exome Variant Server (14), and ATP6AP2Ae4 transcripts are naturally occurring splice isoforms in the human brain (13). Furthermore, 2 missense mutations in exon 3 have recently been shown to be responsible for a glycosylation disorder with liver disease, immunodeficiency, and cutis laxa, and patients show signs of psychomotor impairments (15). In another family, 2 affected siblings suffering from either XLID or parkinsonism have been described carrying a putative splice site mutation in ATP6AP2 $($ c.168+6T $>A)$, downstream of exon 2. However, the functional relevance of this putative variant to splicing and ATP6AP2 function remains to be studied (16). To date, the role of ATP6AP2 in development and function of the mammalian CNS remains largely unknown. First studies of neuron-specific Atp6ap2-knockout mice suggested its involvement in central angiotensin II formation and salt-induced hypertension (17). However, the blood pressure in XLID patients carrying ATP6AP2 (c.321C > T, p.D107D) was normal (18). Another study in mice carrying a conditional postnatal Atp6ap2 deletion in postmitotic neurons has identified autophagy deficits associated with impairments of synaptic plasticity, myelination, and memory functions (19).

Here, we identified a new ATP6AP2 variant resulting in expression of more than $80 \%$ ATP6AP2 $\triangle e 4$ in a patient with XLID and fulminant early postnatal neurodegeneration. We assessed the underlying cellular and molecular mechanisms using patient-derived 

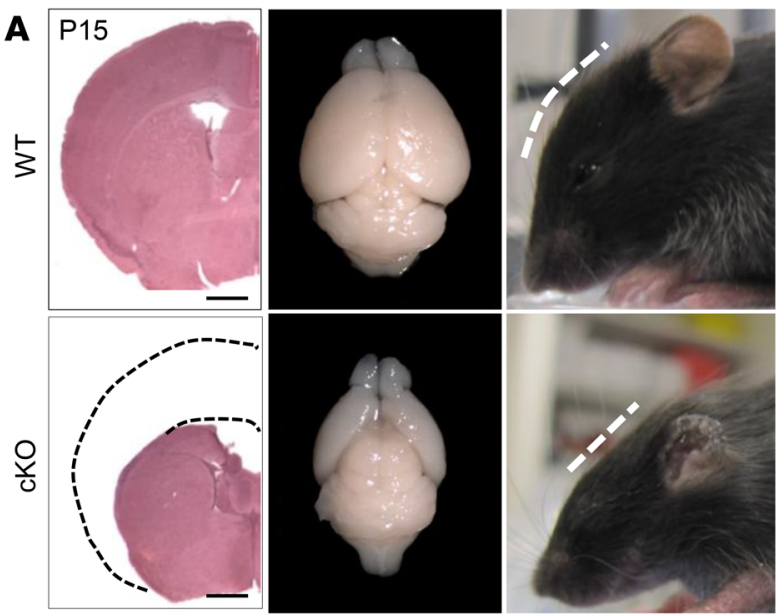

B

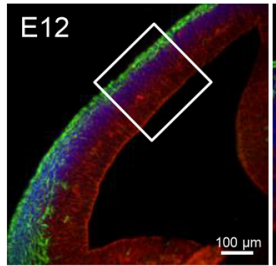

Tuj1 Atp6ap2 DAPI

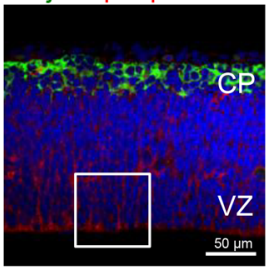

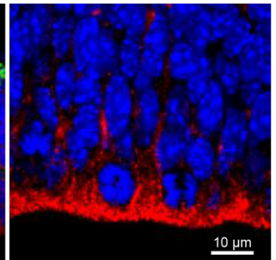
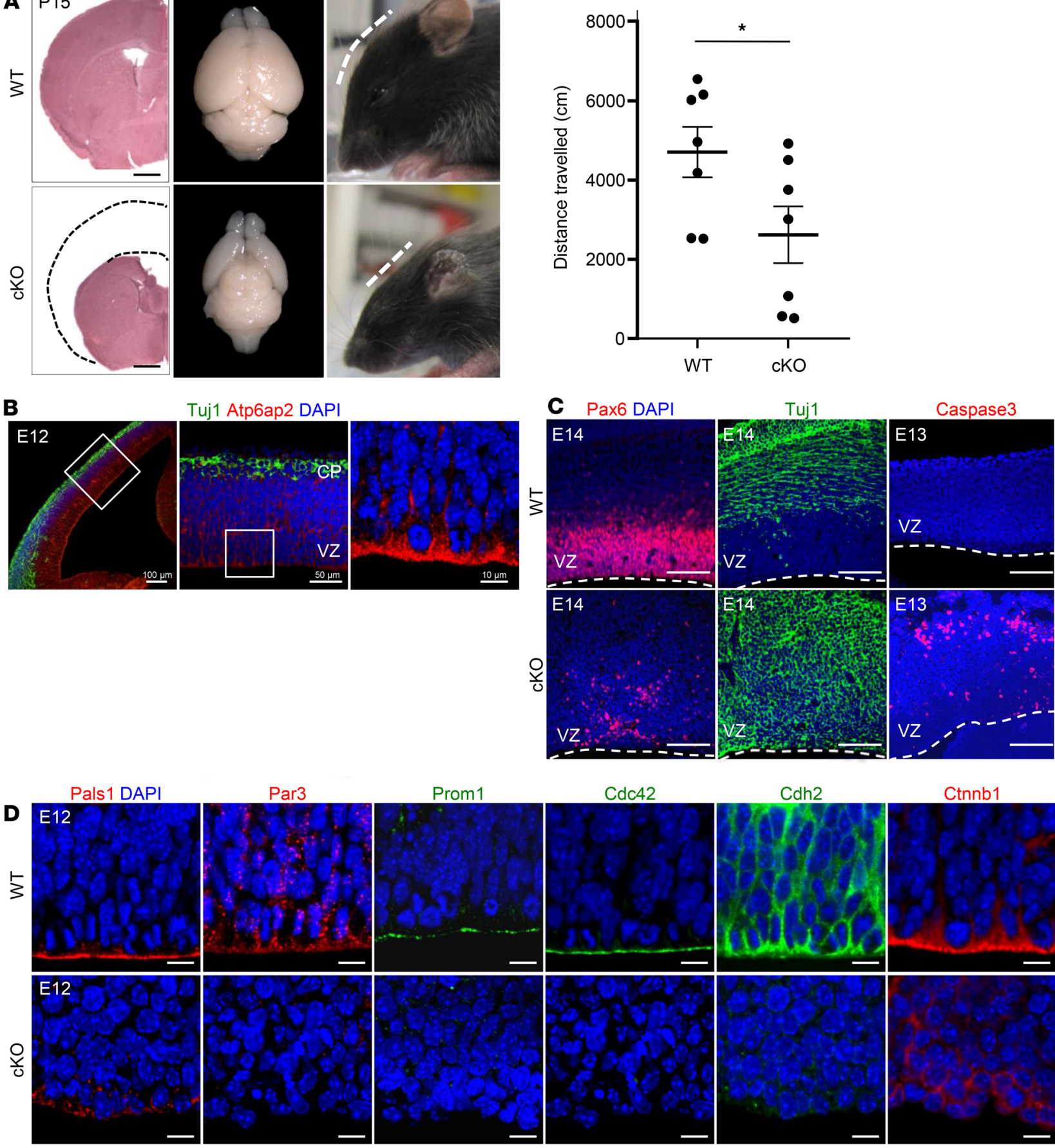

Cdh2

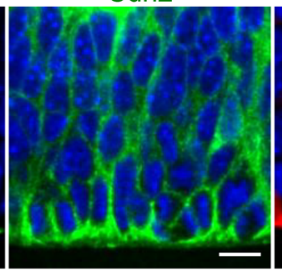

Ctnnb1

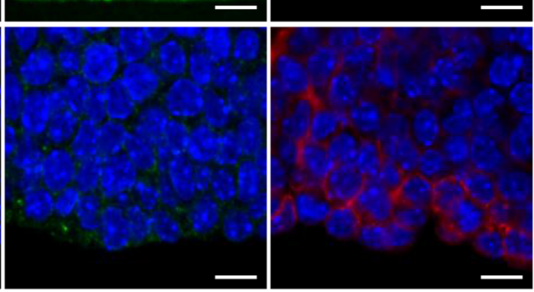

Figure 3. Atp6ap2 is required for cortical development. (A) Left panel: Absence of dorsal cortical structure in cKO mice at P15 evidenced by H\&E staining (left; dashed lines indicate the cortical structure in wild type), brain dorsal view (middle), and flat forehead of mutants (right). Scale bars: $1.0 \mathrm{~mm}$. Right panel: Hypolocomotion in the open field in 15-day-old Atp6ap2lox/y Emx1-Cre-cKO mice. Data show individual values and mean \pm SEM; $n=7$ different animals per group. ${ }^{*} P<0.05$, Student's $t$ test (unpaired, 2-tailed). (B) Atp6ap2 immunofluorescence in E12 cortex shows high expression in radial glial cells (RGCs). VZ, ventricular zone; CP, cortical plate. Scale bars: $100 \mu \mathrm{m}$ (left), $50 \mu \mathrm{m}$ (middle), $10 \mu \mathrm{m}$ (right). (C) Premature neuronal differentiation in cKOs: decrease of Pax6 $6^{+}$RCCs and presence of Tuj1+ cells in the VZ. Apoptosis of newborn neurons shown with activated caspase-3. Dashed lines: ventricular surface. Scale bars: $100 \mu \mathrm{m}$. (D) Reduction of apical complex and adherens junction proteins in E12 cKOs. Scale bars: $10 \mu \mathrm{m}$.

induced pluripotent stem cells (iPSCs) and provide converging evidence from Atp6ap2-conditional-knockout mouse lines and knockdown experiments. Our results show that V-ATPase functions in the developing CNS are critically dependent on the dosage of ATP6AP2.

\section{Results}

Rapidly progressing cerebral atrophy in a patient carrying an ATP6AP2 splice site variant. We identified a de novo intronic $A T P 6 A P 2$ variant in a boy with XLID and fulminant early post- 
natal neurodegeneration (patient 1 ). The patient was diagnosed at the age of 2 weeks with seizures and mild facial dysmorphism, with a short forehead and bitemporal narrowing. At 1 month his occipito-frontal head circumference was at the mean, but it decreased to $\leq 1.5$ standard deviations (SDs) at 6 months and $\leq 2$ SDs at 3 years. He developed intractable seizures and generalized limb spasticity. Sequential MRI at 5 weeks, 9 months, and 28 months of age showed rapidly decreasing cortical gray and white matter volumes, a thin, poorly developing corpus callosum, and myelination deficits (Figure 1A). Candidate gene sequencing revealed a deletion of 2 conserved nucleotides in intron 3 in ATP6AP2 (c.301-11_301-10delTT) (Figure 1, B and $C)$, a variant not present in genome databases of normal genetic variation in human populations. Furthermore, Sanger sequencing of flanking exons and introns (3-5) and the putative promoter region of ATP6AP2 did not reveal additional abnormalities. Human Splicing Finder (20) analysis predicted that the deletion disrupts a branch point motif (CTCTTAA) at position c.30114 expected to increase exon 4 skipping. Accordingly, reverse transcription PCR (RT-PCR) of patient fibroblasts showed 20\% full-length ATP6AP2 (fl-ATP6AP2) and 80\% ATP6AP2 $4 e 4$ transcripts (Figure 1, D and E).

In addition, we performed MRI on a patient from the previously reported family (patient 2, proband IV-18; ref. 18) diagnosed with seizures and moderate mental retardation carrying ATP6AP2 variant (c.321C>T, p.D107D; Figure 2I) resulting in about 50\% ATP6AP2 $\triangle$ e 4 transcripts (12). MRI at the age of 15 revealed an abnormally thin corpus callosum and diffuse volume loss of cortical gray and white matter, although to a substantially lesser degree than in patient 1 (Figure $2, \mathrm{~A}-\mathrm{H}$ ). These results suggested neurodevelopmental deficits and/or neurodegenerative processes that prominently affect cortical regions in these patients.

Atp6ap2 is required for cortical development. We therefore studied neural Atp6ap2 functions in cortical development. We first analyzed its cortical expression in embryonic day 12 (E12) mice. Atp6ap2 was detectable throughout the developing cortex with notable apical enrichment in radial glial cells (RGCs) along the ventricular surface (Figure 3B). Characteristically RGCs are polarized, displaying a specialized apical membrane domain separated from the basolateral domain by adherens junctions. Apical-basal polarity is crucial for the distribution of cell fate determinants during RGC mitosis to balance the generation of progenitors and neurons (21). The subcellular distribution of Atp6ap2 therefore may suggest its involvement in RGC polarity and ultimately cell fate choice, consistent with recent data in retinal progenitors, suggesting that Atp6ap2 interacts with polarity complex protein Par3 (partitioning defective 3 homolog) and that Atp6ap2 knockout disrupts the development of retinal lamination (22).

To assess Atp6ap2 functions in corticogenesis, we generated mutant mice carrying a conditional deletion (Atp6ap2 ${ }^{\text {lox/y }}$ Emx1$\mathrm{Cr}^{+/-}$). In mutant male offspring (Atp6ap2 mutant or conditional knockout [cKO]), protein expression was undetectable by E12 (Supplemental Figure 1A; supplemental material available online with this article; https://doi.org/10.1172/JCI79990DS1). Mice were born at the expected Mendelian ratio, although mutant males showed a postnatal growth delay and died around 4 weeks of age. They displayed a flattened forehead corresponding with a lack of nearly their entire cortex (Figure 3A), suggesting severe deficits in neural cell generation and/or survival. Immunostaining showed a substantial reduction of the transcription factor Pax6 (Figure 3C), essential for RGC proliferation and self-renewal (23). To study proliferation and cell cycle reentry, we performed single-pulse BrdU labeling at E12 and analyzed BrdU ${ }^{+} \mathrm{Ki} 67^{+}$double-labeled cells at E13. In mutants, the number of $\mathrm{Ki}^{+} 7^{+}$cells and the ratio of $\mathrm{BrdU}^{+} \mathrm{Ki} 67^{+}$cells over total $\mathrm{BrdU}^{+}$cells was significantly lower than in controls, indicating reduced proliferation and increased cell cycle exit of progenitors (Supplemental Figure 1C). Correspondingly, the early neuronal marker Tuj1 was ectopically expressed in proliferative zones of the developing cortex (Figure 3C). Subsequently, these prematurely differentiating neurons underwent apoptosis as indicated by activated caspase-3 immunolabeling (Figure $3 \mathrm{C}$ and Supplemental Figure 1D).

The premature progenitor differentiation and subsequent fulminant death of newborn neurons resembled observations in mice carrying a conditional deletion of Pals1 (protein associated with Lin7), encoding an apical complex protein. Rescue experiments in Pals1 mutants suggested a role for mTOR kinase signaling in coupling apical complex dysfunction in progenitors and neuronal cell death (24).

We studied the expression of proteins involved in RGC polarity in Atp6ap2 mutants. Immunolabeling revealed that Pals1, Par3, Prom1 (prominin, CD133), or Cdc42, and adherens junction proteins Cdh2 (N-cadherin) and Ctnnb1 ( $\beta$-catenin), were largely absent in Atp6ap2 cKO (Figure 3D, Supplemental Figure 1B, and Supplemental Figure 2). The failure to detect a broad range of apical proteins in Atp6ap2 cKOs suggested severe deficits in fundamental cellular processes such as protein trafficking, recycling, or degradation interfering with multiple signaling pathways. This is supported by studies in mice with conditional Atp6ap2 deletion in postnatal neurons (19), cardiomyocytes (9), or podocytes $(10,25)$ showing impaired autophagy and increased cell death.

Lysosomes are terminal compartments of endocytosis and autophagy and are signaling hubs to coordinate cellular responses to changes in nutrient availability via mTOR signaling (26). We studied the involvement of Atp6ap2 in lysosomal functions in the developing cortex. We first assessed the subcellular localization of Atp6ap2 in RGCs using super-resolution stimulated emission depletion (STED) imaging on E12 wild-type cortical slices. Atp6ap2 was largely undetectable at the plasma membrane and was mostly found in intracellular punctae (Supplemental Figure 3), suggesting main vesicular functions. Double immunolabeling of Atp6ap2 with lysosomal-associated membrane protein 1 (Lamp1) or with the small GTPase Rab5 or Rab7 showed partial costaining indicating its presence, particularly in lysosomes and in early and late endosomes, respectively (Supplemental Figure 3). Lysosome positioning has been shown to regulate autophagic flux and mTOR signaling. With nutrients available, lysosomes are preferentially located at the cell periphery close to its upstream signaling elements and recruit and activate mTORC1. During starvation, lysosomes cluster in the perinuclear area and mTORC1 signaling is inhibited (27). Confocal imaging of Lamp1 in Atp6ap2 mutants revealed enlarged lysosomes clustered in the perinuclear region, whereas lysosomes in controls were mostly distributed more apically (Figure 4A). Consistent with the perinuclear positioning of lysosomes in mutants, immunolabeling revealed decreased mTOR phosphorylation similar to starvation 
A
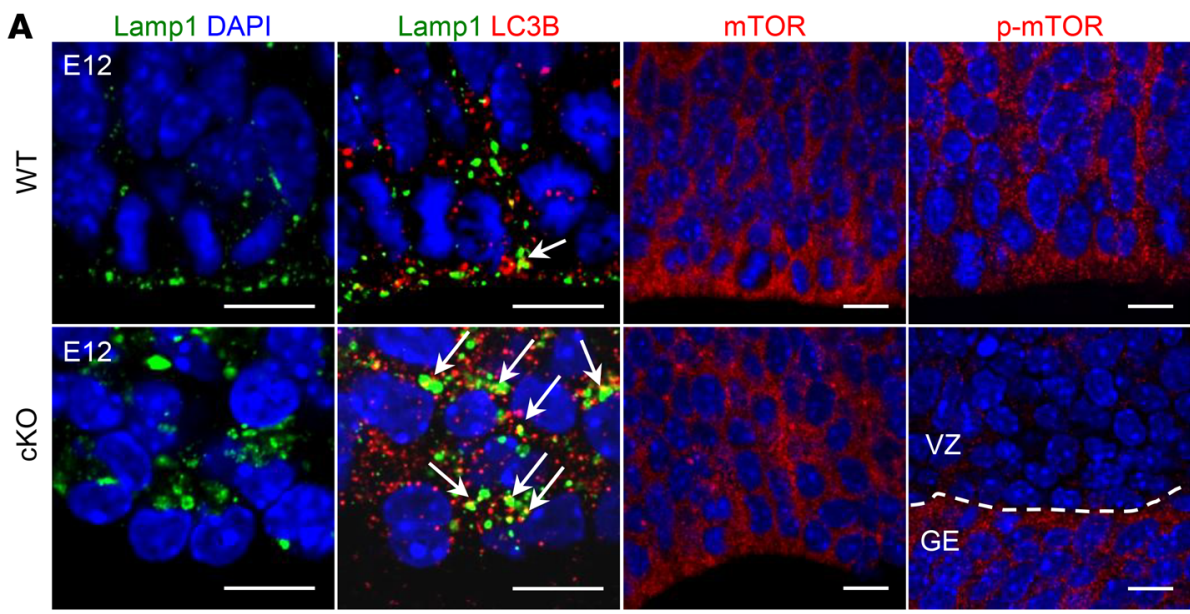

B

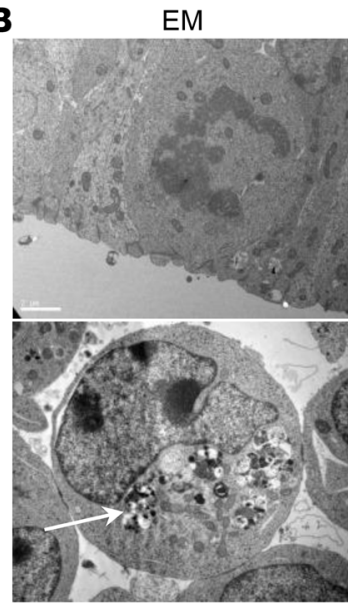

C
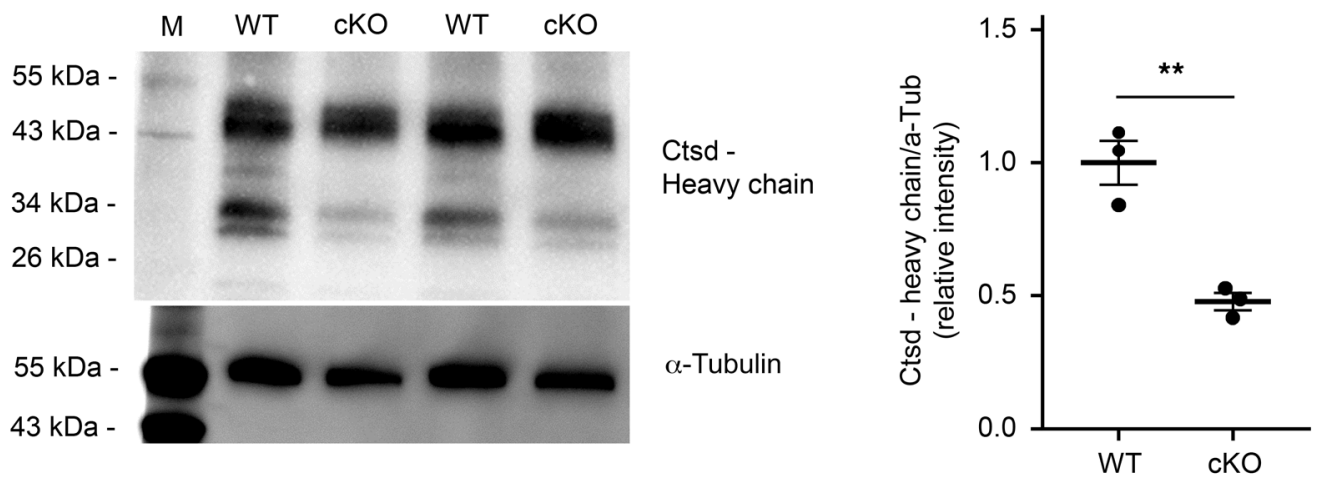

Figure 4. Atp6ap2 is required for lysosomal functioning in cortical development. (A) Immunofluorescence stainings at E12. Left: Lamp1 (lysosomalassociated membrane protein 1) shows enlarged lysosomes with perinuclear clustering in the cKO cortex. Increased number of LC3B (microtubule-associated light chain 3B) aggregates in cKO mice and their colocalization with Lamp1 (arrows). Scale bars: $100 \mu \mathrm{m}$. Right: While mTOR levels appear normal in cKOs, there is a marked reduction of phospho-mTOR (p-mTOR). Since Atp6ap2 deletion in cKOs is restricted to the dorsal cortex, p-mTOR in the ganglionic eminence (CE) in mutants is normal. Scale bars: $10 \mu \mathrm{m}$. (B) Electron microscopy (EM) shows large vacuolar structures with dense material (arrow). (C) Cathepsin D (Ctsd) Western blot on E12 cortex. The proenzyme of the cathepsin protease is proteolytically processed in the lysosome, which requires strongly acidic $\mathrm{pH}$ to yield the $34-\mathrm{kDa}$ fragment. This processing is disturbed in the cKO cortex. Data show individual values and mean $\pm \mathrm{SEM} ; n=3$ different animals per group. ${ }^{* *} P<0.01$, Student's $t$ test (unpaired, 2 -tailed).

conditions (Figure 4A and Supplemental Figure 4A). Starvation or lysosomal stress has been shown to promote lysosomal biogenesis, autophagosome formation, and fusion of autophagosomes with lysosomes (3). In mutants but not in controls, we detected increased punctae positive for the autophagosomal membrane protein LC3B that partially overlapped Lamp1 (Figure 4A and Supplemental Figure 4). Electron microscopy in cortical progenitors revealed large, electron-dense vacuolar structures (Figure 4B). These results indicate induction of autophagy and accumulation of autophagosomes but deficient protein degradation in autophagolysosomes in mutants. This is further supported by cathepsin D Western blots on embryonic cortical tissue. The activation of this protease requires proteolytic processing in lysosomes and is $\mathrm{pH}$ dependent. Our results indicate that cathepsin D processing is impaired in cKOs (Figure 4C).

Taken together these results suggest that Atp6ap2 is a key regulator of V-ATPase functions during corticogenesis. Atp6ap2 deficiency may lead to dysfunctions of several V-ATPase-dependent cellular processes such as vesicular acidification, protein degradation, and mTOR signaling.
Postnatal neuronal knockout of Atp6ap2 impairs protein degradation and causes memory deficits. Such ATP6AP2-dependent V-ATPase dysfunctions may also lead to neurodegenerative processes at later developmental stages or in adulthood, as suggested by the association of ATP6AP2 variant c.345C $>$ T with XPDS (13). Therefore we studied mice carrying an Atp6ap2 deletion in postmitotic neurons in the forebrain including hippocampus and cortex starting around 1 month of age (Atp6ap2 $2^{l o x / y} \mathrm{Cam}$ $\left.\mathrm{K} 2 \mathrm{a}-\mathrm{Cr} \mathrm{e}^{+/}\right)$. Around 4 months of age, mutants developed pathological hind-limb clasping reflexes during tail suspension (Figure $5 \mathrm{~A}$ ) and hyperactivity in the open field (Figure 5B), potentially representing early signs of neurodegeneration $(28,29)$. We furthermore assessed long-term memory function using a hippocampusdependent contextual fear-conditioning task (30). We found that 4-month-old mutants were indistinguishable from control littermates whereas at 6 months they displayed significantly impaired long-term memory (Figure 5C), suggesting a progression of neural dysfunction reminiscent of other neurodegenerative disorders. To test whether behavioral abnormalities were correlated with mor- 
A

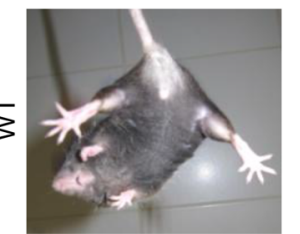

B

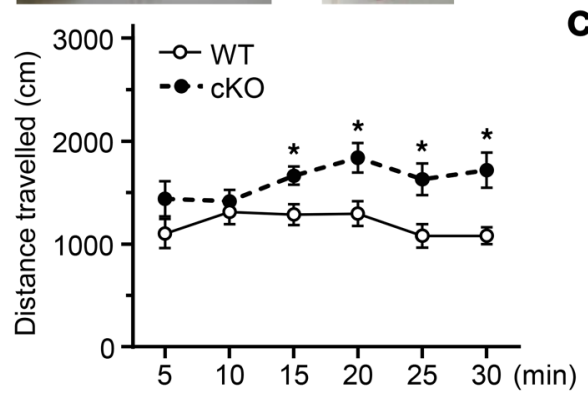

D

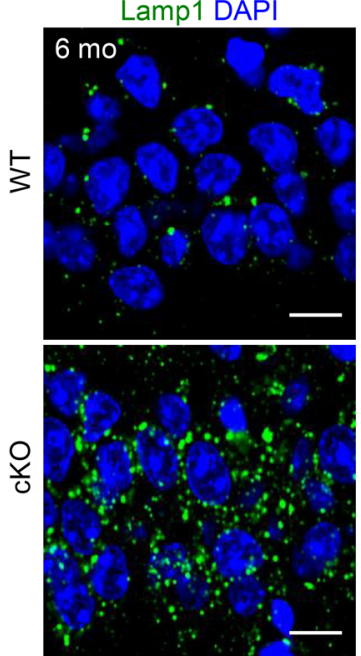

E
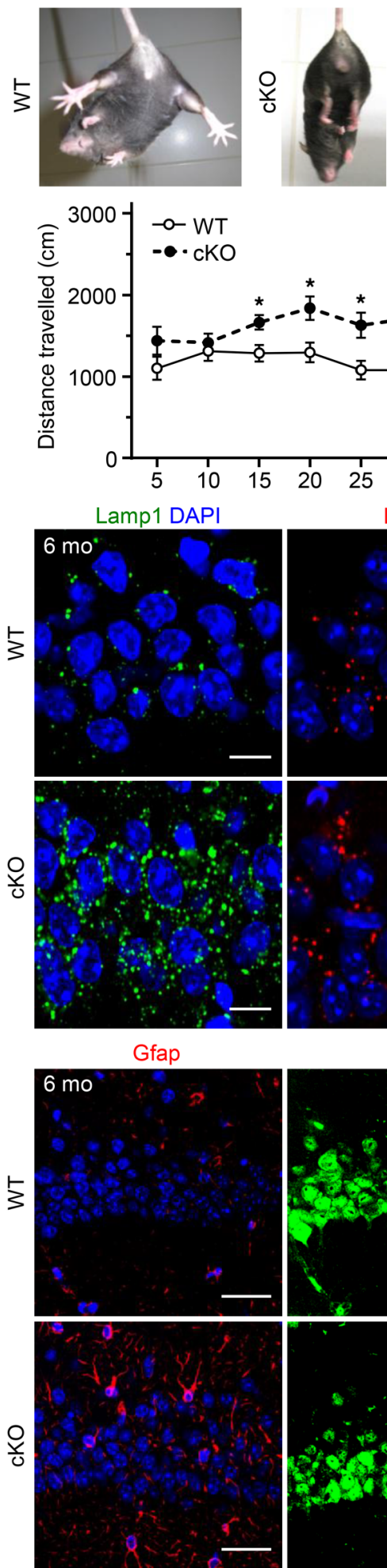

NeuN
C
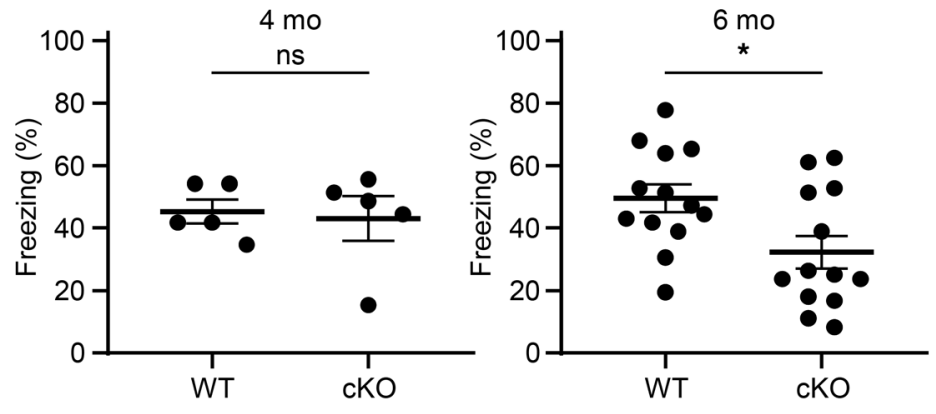

p62
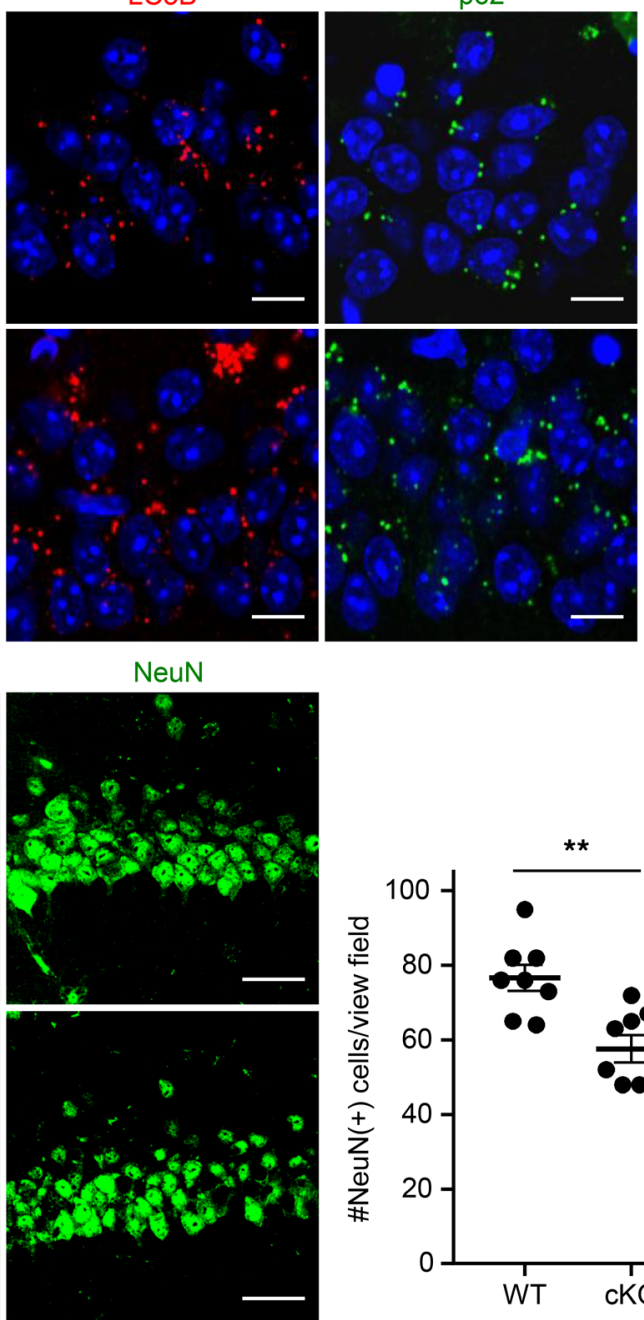

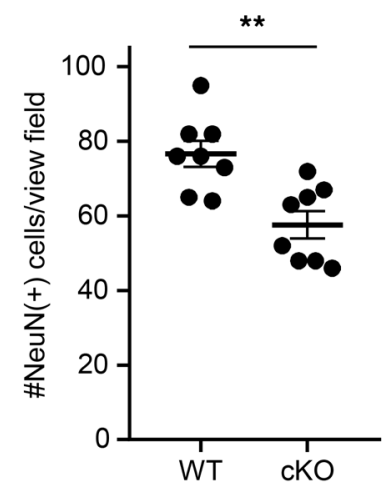

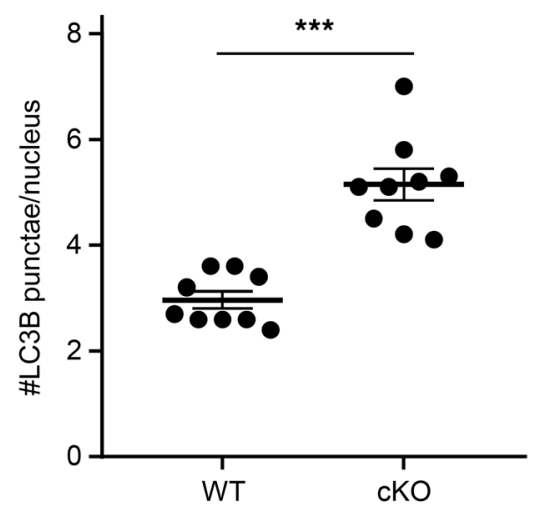

$\mathbf{F}$

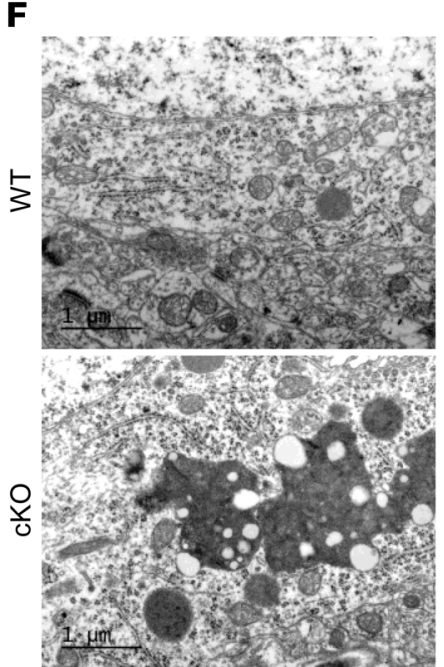

Figure 5. Postnatal Atp6ap2 deletion in postmitotic neurons causes behavioral deficits and accumulation of protein aggregates. (A and B) Abnor-

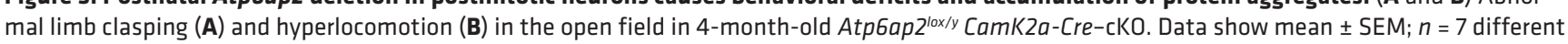
animals per group. ${ }^{*} P<0.05$, 2-way repeated-measures ANOVA followed by Bonferroni's post test. (C) Deficient long-term memory in 6 -month-old ( $n=$ 13 different animals per group; right) but not in 4-month-old ( $n=5$ different animals per group; left) cKO mice. Data show individual values and mean \pm SEM; ${ }^{*} P<0.05$; ns, $P>0.05$; Student's $t$ test (unpaired, 2-tailed). (D) Immunostaining in CKOs shows enlarged lysosomes and accumulation of LC3B and p62 aggregates in the hippocampus (CA1). Scale bars: $10 \mu \mathrm{m}$. Right: Quantification of LC3B ${ }^{+}$punctae per nucleus. Data show individual values and mean $\pm \mathrm{SEM} ; n=6$ different animals per group. ${ }^{* *} P<0.001$, Student's $t$ test (unpaired, 2-tailed). (E) Left: Gfap (glial fibrillary acidic protein) immunofluorescence is increased in the cKO CA1 hippocampus. Right: Decreased number of NeuN ${ }^{+}$neuronal nuclei in the CA1 hippocampus in 6-month-old cKO mice. Data show individual values and mean $\pm \mathrm{SEM} ; n=8$ different animals per group. ${ }^{* *} P<0.01$, Student's $t$ test (unpaired, 2 -tailed). Scale bars: $50 \mu \mathrm{m}$. (F) Electron microscopy shows accumulation of electron-dense material in hippocampal CA1 neurons in cKO mice. 
phological signs of lysosomal pathway dysfunction, we performed immunostaining on hippocampus in 6-month-old mutants. Similar to observations in the embryonic brain, Lamp1 staining revealed an increased accumulation of enlarged lysosomal compartments clustered in the perinuclear region (Figure 5D). We further examined autophagosomes by staining for LC3B and its interacting partner p62/SQSTM1 that targets ubiquitinated proteins to autophagosomes. We found increased perinuclear staining and a significantly higher number of $\mathrm{LC}^{+}$and $\mathrm{p} 62 / \mathrm{SQSTM} 1^{+}$punctae (Figure 5D and Supplemental Figure 5). While we did not detect fulminant neuronal degeneration as measured by an increased immunoreactivity of the apoptosis marker activated caspase-3 (data not shown), immunostaining for glial fibrillary acidic protein (Gfap) suggested an upregulation and hypertrophic astrocytic processes as signs of gliosis typically associated with neurodegenerative processes. This was further supported by a reduced number of hippocampal neurons in the CA1 region (Figure 5E). Moreover, electron microscopy studies revealed an accumulation of electron-dense material in the same region (Figure 5F). Collectively, these data demonstrate that mature as compared with developing neurons are less sensitive to Atp6ap2 deficiency and undergo a protracted neurodegenerative process.

Abnormal "in vitro corticogenesis" of patient iPSC-derived neurons. To study whether increased exon 4 skipping causes lysosomal dysfunction during human cortical development, we generated iPSCs from patient 1 (Supplemental Figure 6). To provide further proof of causality of ATP6AP2 44 for observed phenotypes, we generated an isogenic control line by stable re-expression of fulllength ATP6AP2 (fl-ATP6AP2) in the patient iPSCs (Supplemental Figure 6F). When sequentially cultured in media containing different growth factors, iPSCs recapitulate key steps of in vivo corticogenesis (31). Control iPSCs organized after 20 days in vitro (DIV) into characteristic sphere-like structures termed neural rosettes resembling the cortical neuroepithelium, with $\mathrm{PAX}^{+}$progenitors at the luminal surface and $\mathrm{TUJ} 1^{+}$neurons in the outer layer (32). Patient-derived neural rosettes, however, showed TUJ1 ${ }^{+}$cells interspersed in the $\mathrm{PAX}^{+}$progenitor zone, suggesting premature and/or ectopic differentiation (Figure 6B). Western blots of iPSCs and their derived neuronal progenitors showed an upregulation of LC3B and p62, indicating that altered autophagy activity is already present at the earliest developmental stages (Supplemental Figure 7). We used the acidotropic dye LysoSensor Yellow/Blue dextran to quantify vesicular acidification across a broad (3.0-9.0) $\mathrm{pH}$ range (33). LysoSensor measurements showed a significantly increased mean vesicular $\mathrm{pH}$ in patient iPSC-derived cortical neurons suggesting an impaired V-ATPase function (Figure 6C). Associated lysosomal dysfunction was further supported by impaired proteolytic processing of cathepsin D in patient-derived progenitors as assessed in Western blots (Supplemental Figure 8). However, the observed phenotypes did not impair the proliferation potential of patient iPSC-derived progenitor cells as assessed by PAR3, CDH2, and CTNNB1 expression and phospho-histone 3 immunolabeling (Figure 6A).

Impaired proteostasis, activity, and survival of patient iPSCderived neurons. We further studied early developing neurons. Electron microscopy of these neurons showed excessive accumulation of large vacuoles containing diverse material resembling late autophagic compartments and increased lysosomal diameter (Figure 7A). With progressing time of neuronal differentiation, cell death of patient iPSC-derived neurons significantly increased in comparison with controls, as confirmed by activated caspase-3 immunostaining (Figure 7B). The survival of developing cortical neurons is critically dependent on early spontaneous activity (34). We measured spontaneous intrinsic neuronal activity in developing iPSC-derived cortical neurons at 45 DIV using calcium imaging (35). We found that the number of spontaneously active neurons as well as the frequency of their activity was significantly lower in patient than in control iPSC-derived cultures (Figure 8). Thus ATP6AP2 deficiency may impair early spontaneous activity, which in turn may contribute to the fulminant neurodegeneration observed in the patient (36).

ATP6AP2 $\triangle$ e4 impairs V-ATPase activity and functions. In order to gain further insights into ATPase dysfunctions due to the lack of the exon 4-encoded ATP6AP2 domain, we studied HeLa cells using siRNA-mediated knockdown yielding approximately $75 \%$ reduction of protein levels (Supplemental Figure 9, A and B). LysoSensor assays showed that ATP6AP2 knockdown increased median vesicular $\mathrm{pH}$ from 6.07 to 6.54 with a notable decrease of the more acidic vesicles ( $\mathrm{pH} 3.5-4.5)$ likely representing lysosomes. Cotransfection with fl-ATP6AP2 but not with ATP6AP2 $\triangle \mathrm{e} 4$ restored $\mathrm{pH}$ to control levels, confirming that the exon 4-encoded domain is necessary for vesicular acidification (Supplemental Figure 9C), consistent with previous results (37). Overexpression of ATP6AP2 in HeLa cells did not change vesicular pH (Supplemental Figure 9D). Furthermore, ATPase activity in lysosomal fractions of HEK293T cells was significantly reduced following ATP6AP2 knockdown and could be restored by cotransfection with fl-ATP6AP2 but not with ATP6AP2 $\triangle \mathrm{e} 4$ (Supplemental Figure 10).

We next assessed whether this acidification deficit is associated with impaired degradation of endocytosed proteins. We analyzed ligand-induced degradation of epidermal growth factor receptor (EGFR) (38) using a HeLa cell line with stable ATP6AP2 knockdown and found that ATP6AP2 knockdown causes delayed decay of EGFR (Supplemental Figure 11). Although decreased activity of lysosomal hydrolases due to impaired vesicular acidification may account for this delay, inhibition of V-ATPase activity may also impair endosomal-lysosomal fusion (39). We used time-lapse confocal microscopy to study fusion events following ATP6AP2 knockdown. LysoTracker Red was used to label lysosomal compartments, whereas endosomes were identified via uptake of a pulse of Oregon green 488 dextran; the subsequent appearance of yellow dots indicated fusion events. Whereas control cultures displayed a characteristic combination of "kissing" and fusion events, ATP6AP2 knockdown surprisingly caused a rapid and significant increase in fusion events especially in large perinuclear clusters. Cotransfection with fl-ATP6AP2 but not with ATP6AP2 $\triangle \mathrm{e} 4$ restored fusion events to control levels (Figure 9, A and B, and Supplemental Videos 1-4). Several recent studies have suggested that the VO sector of the V-ATPase acts in vesicular membrane fusion independently from its function as proton pump, although this is still debated (40-48). In any case, both lower proton pump activity of V-ATPases and increased vesicular fusion could be related to decreased membrane content of assembled V0-V1 holoenzyme complex. We assessed a 
A

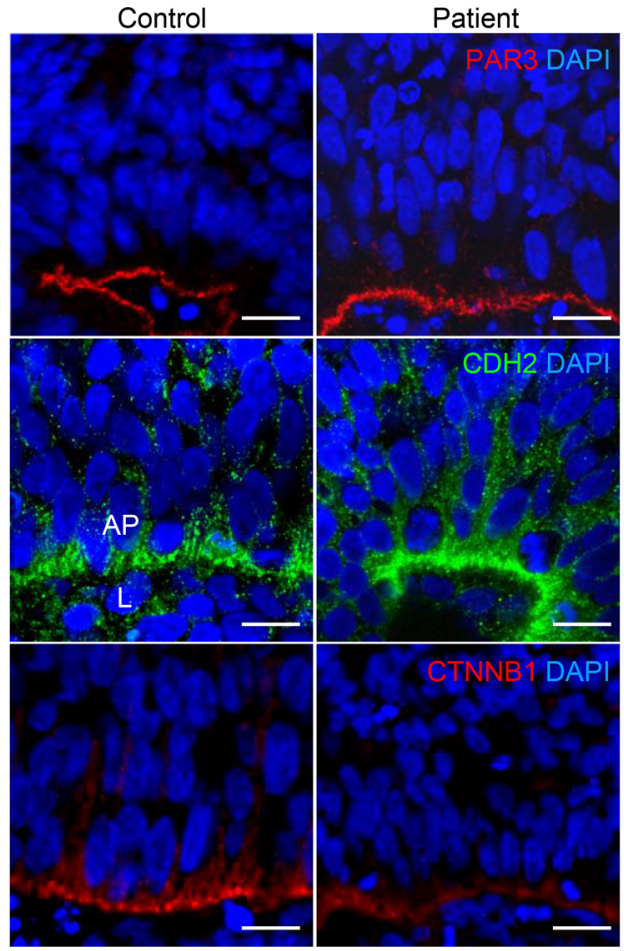

B

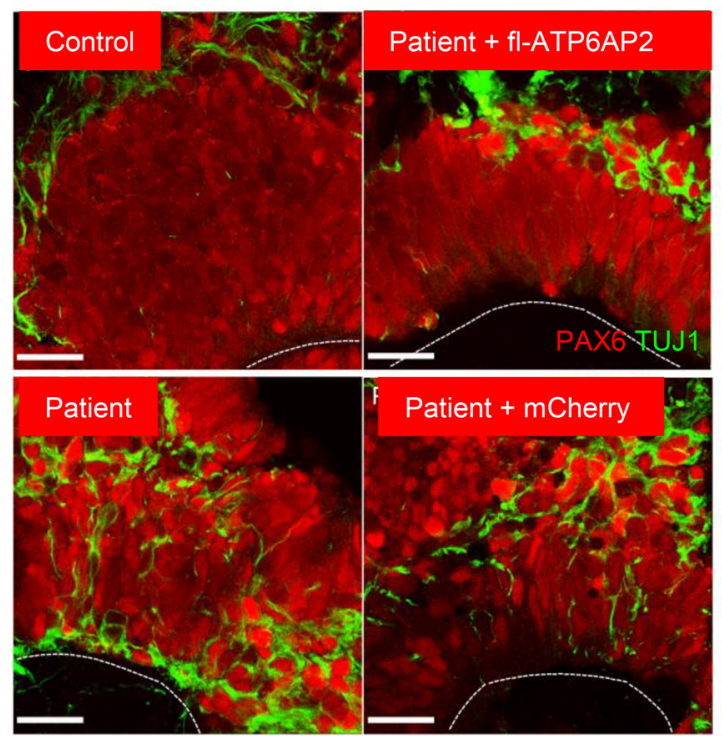

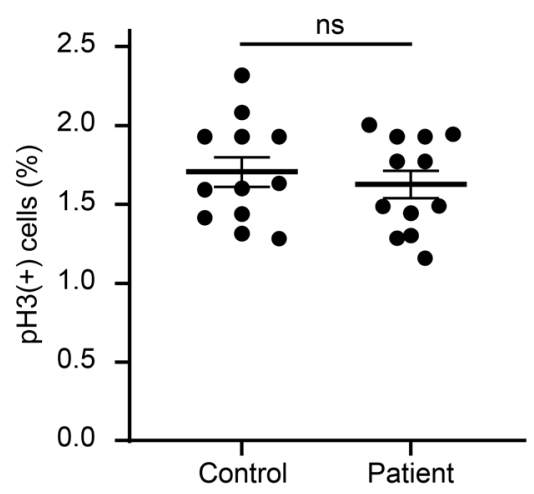

C
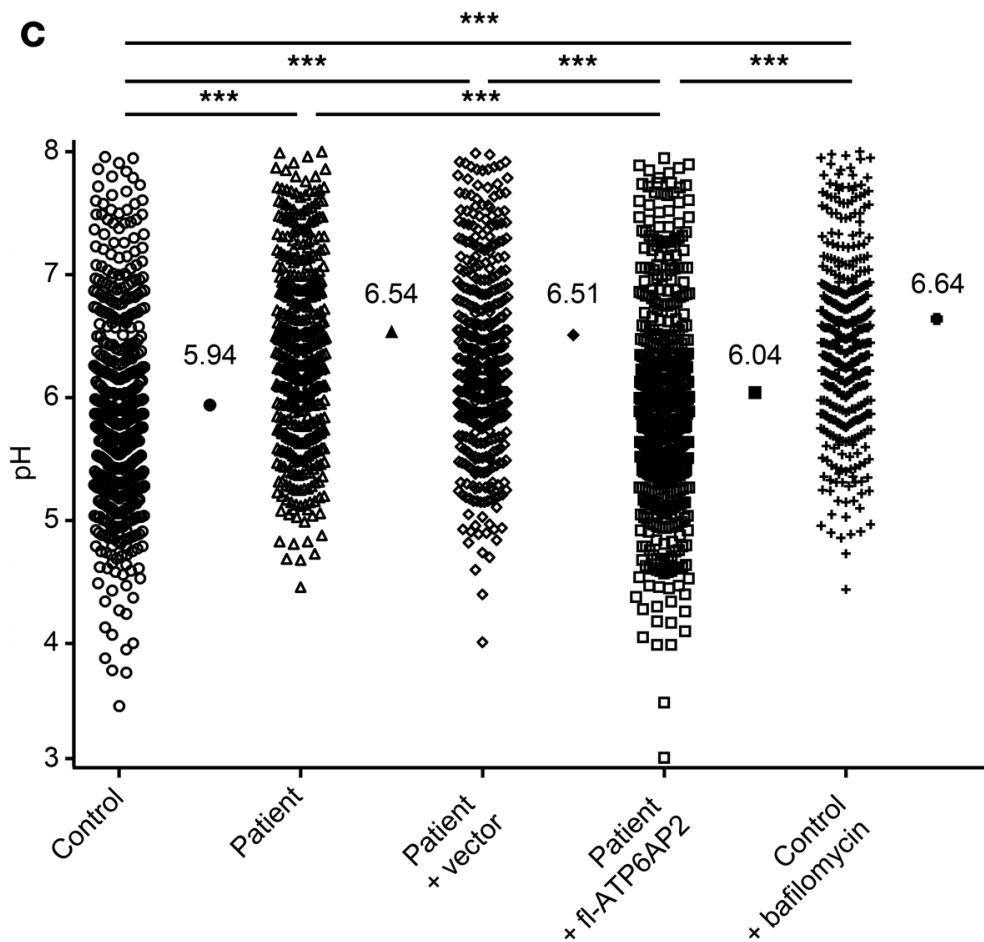

Figure 6. Abnormal "in vitro corticogenesis" of patient iPSC-derived neurons. (A) Left: Laminar organization of proliferative and differentiation compartments of "cortical" rosettes derived from control and patient iPSCs after 20 days in vitro (DIV). Normal expression pattern of apical marker PAR3 and adherens junctions markers CDH2 and CTNNB1. AP, apical pole; L, lumen. Scale bars: $10 \mu \mathrm{m}$. Right: Phospho-histone 3 (pH3) immunostaining did not reveal differences in progenitor proliferation between control and patient. Data show individual values and mean \pm SEM; $n=12$ per group. $P=0.341$ (ns), Student's $t$ test (unpaired, 2-tailed). (B) Immunostaining with PAX6 and TUJ1 shows interspersed neurons in the progenitor zone of patient-derived rosettes. This phenotype is rescued by re-expression of fl-ATP6AP2. Lines: luminal surface. Scale bars: $25 \mu \mathrm{m}$. (C) Significantly increased median pH in patient cells in early differentiating neurons. Data show individual values and median; $n=463-550$ measurements from 5 independent experiments. ${ }^{* * *} P<0.001$, Kruskal-Wallis test $\left(\chi^{2}=245.97, \mathrm{df}=4, P<0.001\right)$ followed by Dunn's multiple-comparisons test. 
A
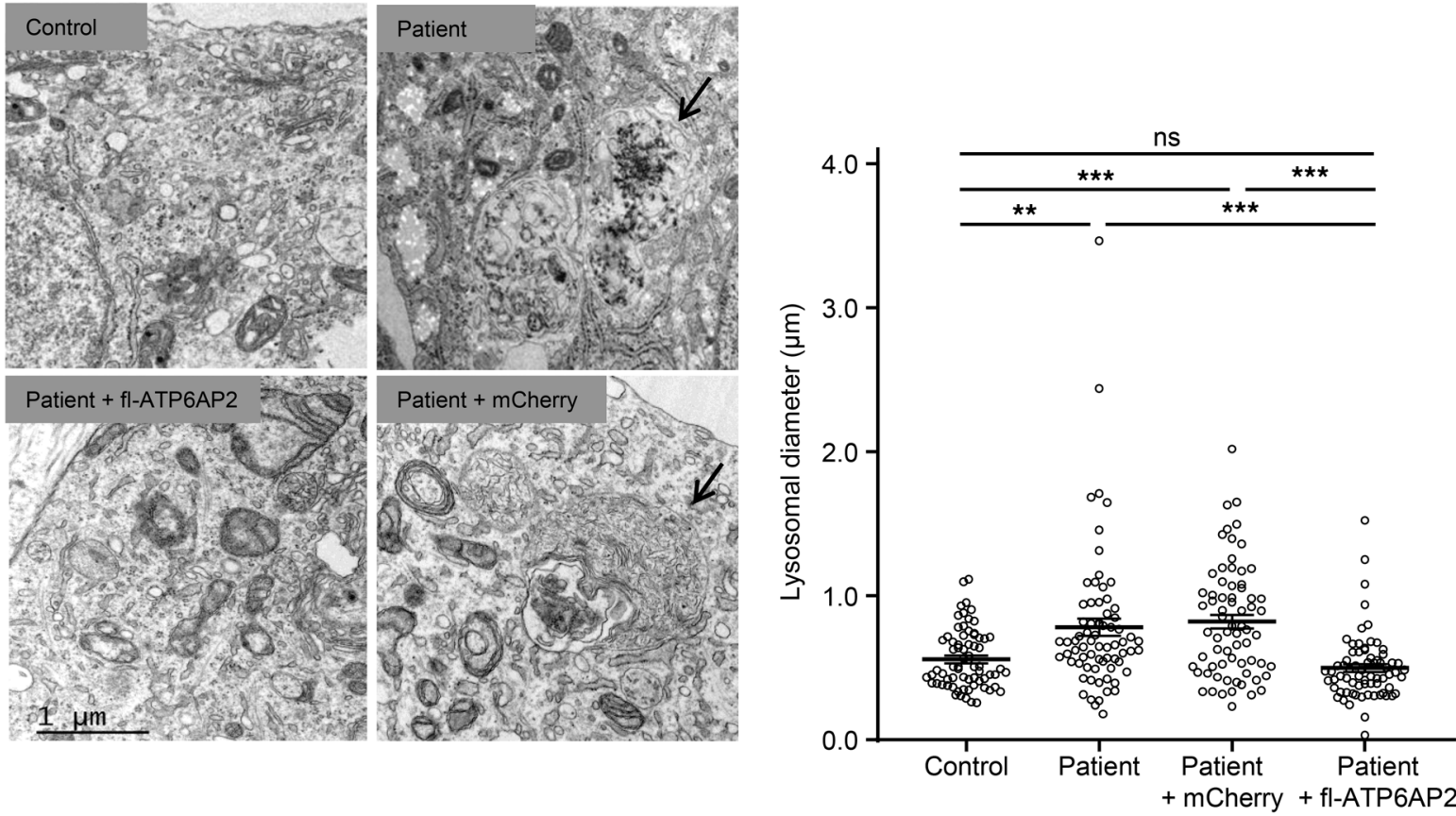

B
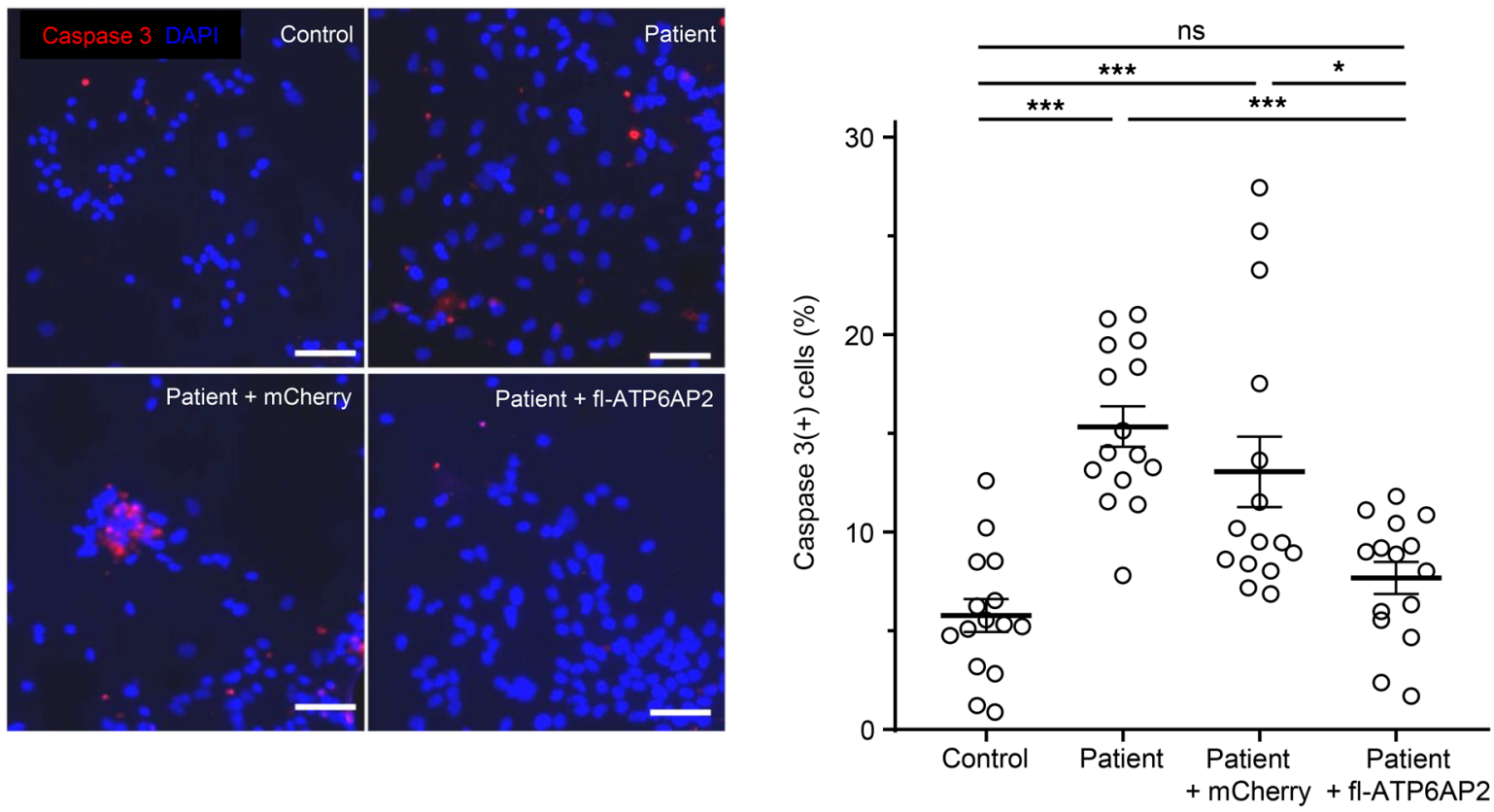

Figure 7. Degeneration of patient iPSC-derived cortical neurons. (A) Left panel: Electron microscopy (EM) of iPSC-derived differentiating neurons (45 DIV) shows excessive vesicles with electron-dense undigested material (arrow, top right) and multilamellar inclusions (arrow, bottom right) resembling late autophagosomal compartments. Scale bar: $1.0 \mu \mathrm{m}$. Right panel: Quantification of lysosomal diameter from EM images. Data show individual values and mean \pm SEM; $n=69-70 .{ }^{* *} P<0.01,{ }^{* *} P<0.001$, Kruskal-Wallis test $\left(\chi^{2}=44.61, \mathrm{df}=3, P<0.0001\right)$ followed by Dunn's multiple-comparisons test. (B) Left: Increased activated caspase-3 immunostaining in patient iPSC-derived cortical neurons. Scale bars: $50 \mu \mathrm{m}$. Right: Positive cells were quantified. Data show individual values and mean $\pm S E M ; n=15$ per group. ${ }^{*} P<0.05$, ${ }^{* * *} P<0.001$, 1-way ANOVA followed by Bonferroni's post test.

potential change in V0-V1 composition by measuring the membrane ratio of the VOa1 subunit that has been involved in neurodegeneration (49) and of the V1B2 subunit highly expressed in the human brain (50). We found that ATP6AP2 knockdown causes a significantly lower ratio of membrane-assembled V1B2-VOa1 as compared with controls. Cotransfection with fl-ATP6AP2 but not with ATP6AP2 $\triangle \mathrm{e} 4$ restored the V1B2-V0a1 ratio to control levels (Figure 9C). Furthermore we were able to confirm these results in patient iPSC-derived neural progenitor cells (Supplemental Figure 12). Taken together these in vitro studies indicate that the lack of the exon 4-encoded ATP6AP2 domain impairs several V-ATPase-mediated functions. 


\section{Discussion}

Here we studied a patient with fulminant neurodegeneration carrying a rare ATP6AP2 splice site mutation. We found that patient iPSC-derived neural cells and targeted Atp6ap2 knockout in the mouse brain converge on common phenotypes. This provides evidence for the causality of observed ATP6AP2 splicing imbalances in this rare disorder and indicates that the complete loss-of-function mouse models have genetic validity to study the involved molecular and cellular mechanisms.

The 2 patients with XLID due to an ATP6AP2/ATP6AP2 4 4 splicing imbalance show a substantially different time course and severity of brain atrophy prominently of cortical gray and white matter. Another synonymous variant in ATP6AP2, c.345C $>\mathrm{T}$ (p.S115S), causing an ATP6AP2/ATP6AP2 4 e4 transcript ratio of approximately 50:50, was reported in a family with XPDS and primarily affected the striatum $(13,51)$. Members of this family displayed a diversity of symptoms, of their severity, and of age of onset (between 14 and 58 years) (51). Immunohistological studies on brain material from one individual who died at age 86 showed the presence of pathological Tau deposits and accumulation of p62/SQSTM1, suggesting defects in protein degradation and autophagy. Although the reasons for the clinical heterogeneity associated with the different ATP6AP2 splice site variants are still unclear, it may involve a specific expression pattern of splicing factors depending on developmental stage and brain region or neural cell type (13). The splicing imbalances clinically manifest as neurodegenerative process along a continuum of ages from early development to adulthood, corresponding with the available amount of fl-ATP6AP2. An ATP6AP2/ATP6AP2 $\triangle$ e 4 transcript ratio of approximately 20:80 ultimately leads to fulminant neurodegeneration in early development, while, in contrast, a transcript ratio of approximately 50:50 may affect primarily the CNS over a period of several years with no clinical symptoms in other organ systems. This suggests that although ATP6AP2 is widely expressed, the CNS is exquisitely dosage sensitive.

The patient iPSC-derived "in vitro corticogenesis" experiments suggest that cell polarity and proliferation potential of cortical stem cells are largely preserved, which is supported by the normal head circumference of the patient around birth. However human ATP6AP2-deficient progenitors appear, at least partly, to differentiate prematurely into neurons, which is consistent with our findings in Atp6ap2-knockout mice. These data are also compatible with V-ATPase dysfunctions in cortical progenitors. Expression of a dominant-negative V-ATPase subunit in vivo reduces endogenous Notch signaling, depletes cortical stem cells, and induces neuronal differentiation (52). In Atp6ap2-knockout mice, we observed the lack of multiple apical enriched proteins in cortical stem cells. Given the multitude of fundamental cellular processes depending on V-ATPase activity, our results suggest severe deficits in protein trafficking, sorting, and recycling rather than dysfunction of a single signaling pathway. For example, the reduced levels of Ctnnb1, a downstream effector of the canonical Wnt signaling pathway, would likely affect cortical stem cell self-renewal (53).

Differences in vulnerability of early developing and mature neurons have been noted in a number of neurodegenerative disorders associated with impaired lysosomal degradation (54). Neu- rons naturally undergo a remarkable developmental transformation as they mature, from susceptibility to inhibition of cell death (55). Thus newborn Atp6ap2-deficient neurons may engage apoptosis pathways normally restricted more to the developmental period. Indeed we found that Atp6ap2 deficiency caused a strong upregulation of activated caspase-3, a cell death pathway that has been shown to decay with increased brain maturation (55). The rapid induction of cell death in knockout mice, however, prevented further studies in early developing neurons. The hypomorphic ATP6AP2 mutation in patient iPSCs allowed generation of early neurons. These cells faithfully recapitulated the V-ATPase deficits and showed reduced early spontaneous neuronal activity, a known contributor to increased cell death susceptibility (34). Thus these iPSCs can be instrumental for further mechanistic studies on neural V-ATPase functions.

Exon 4 encodes a part of the N-terminal extracellular/intravesicular domain (Figure 2, J and $\mathrm{K}$ ), outside of the initially identified V-ATPase interaction domain (56). Studies in transfected HEK293T cells, however, found that the extracellular and transmembrane domains of ATP6AP2 interact with the V-ATPase subunit VOC. Moreover, the interaction of the extracellular domain with Wnt receptors has been shown to be required for Wnt signaling (8). Furthermore, expression of ATP6AP2 $\triangle \mathrm{e} 4$ in Atp6ap2-knockout fibroblasts failed to restore vesicular acidification, LC3-II accumulation, and V-ATPase biogenesis (37). However, it remained unclear whether impaired folding or membrane incorporation of these exogenous truncated protein fragments into the large V-ATPase complex may contribute to these negative outcomes (37). Our studies on patient iPSC-derived neurons are consistent with the above results and provide evidence for the requirement of the exon 4-encoded domain in V-ATPase functions. This encouraged us to perform additional dynamic in vitro studies to explore whether the fulminant time course of neurodegeneration in the patient corresponds with deficiency of V-ATPase-associated functions of the exon 4-encoded domain. We found that ATP6AP2 $\triangle \mathrm{e} 4$ is insufficient to rescue the appearance of perinuclear clusters of fused endolysosomes only 24 hours after ATP6AP2 knockdown (Figure 8A). This suggests a rapid contribution of the exon 4-encoded domain to V-ATPase functioning. Further studies are needed to precisely delineate the potential direct and indirect roles of this domain in the regulation of V-ATPase functions.

Mutations of V-ATPase subunits in model organisms are often associated with severe phenotypes and death (57), hampering more detailed functional studies. In addition, few human disorders due to mutations affecting V-ATPases have been described. They mainly affect subunits conferring tissue specificity or subcellular localization, resulting in specific deficits such as renal tubular acidosis, deafness, or osteopetrosis (1). We found that ATP6AP2 is a key accessory protein for V-ATPase functions in the CNS and is essential for stem cell self-renewal and neuronal survival. Furthermore, the exon 4-encoded domain is involved in modulating V-ATPase activity in the CNS but is not required for ATP6AP2 functions as (pro)renin receptor (12). These findings may have implications beyond this rare XLID for more common disorders.

Collectively the patient data suggest that reduced V-ATPase activity due to ATP6AP2 deficiency may have an interesting safety 
A

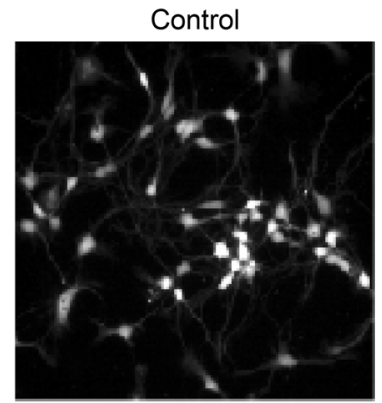

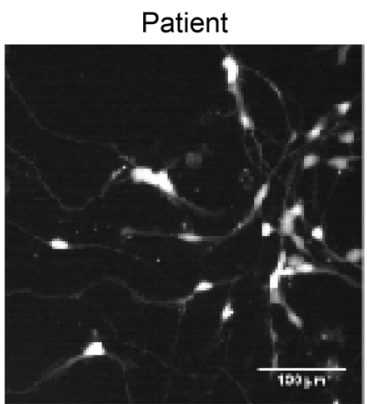
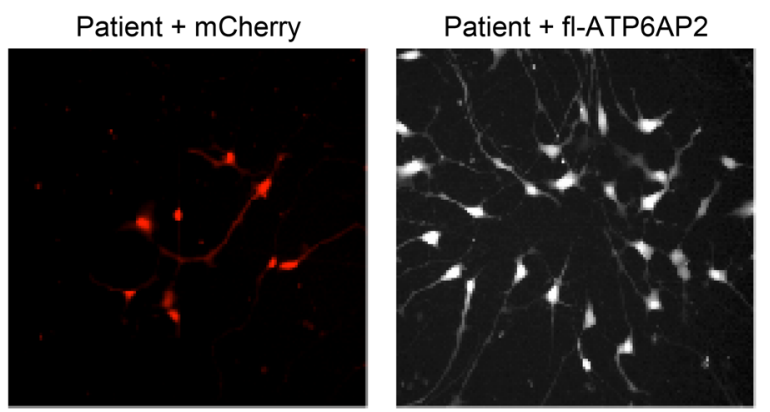

B
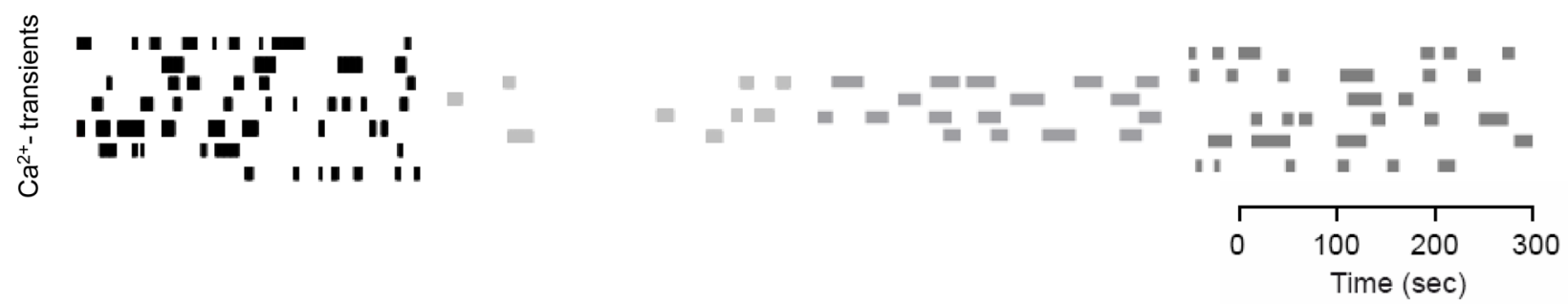

\section{C}
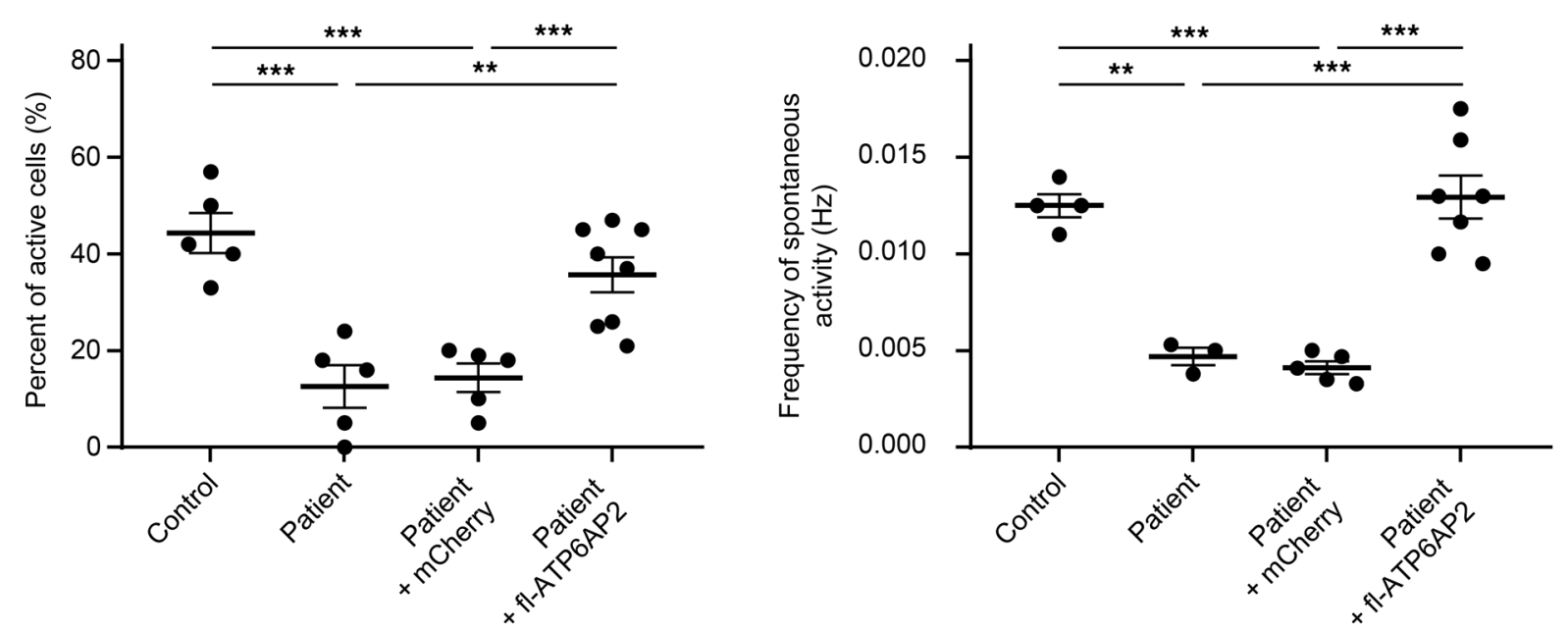

Figure 8. Decreased number of active cells and of calcium transient frequency in patient iPSC-derived differentiating neurons. (A) Representative calcium imaging pictures. (B) Rasterplots of spontaneous calcium transients representing the activity of all neurons in the network. (C) Quantification of active cells (left, $n=5-8$ ) and frequency of spontaneous activity (right, $n=3-7$ ). Data show individual values and mean \pm SEM. ${ }^{* *} P<0.01,{ }^{* * *} P<0.001$, 1-way ANOVA followed by Bonferroni's post test.

margin, affecting the brain while being more tolerated in other tissues. The animal studies suggest that while Atp6ap2 knockout rapidly inhibits stem cell self-renewal, the more mature brain appears less sensitive. This is potentially relevant to the development of V-ATPase inhibitors for brain cancers, which rely heavily on $\mathrm{pH}$ regulation for growth and invasion (58).

Dysfunctions of protein degradation pathways are also a hallmark of common neurodegenerative disorders. Rare genomic variants of major effect size causally linked to common neurodegenerative diseases have pointed to dysfunctions at multiple stages in lysosomal degradation pathways (59). Deficits in particular at late stages of autolysosomal proteolysis due to impaired lysosomal acidification and signaling have been suggested as a trigger for toxic protein accumulations (33). Therefore the identification of V-ATPase-associated molecules regulating V-ATPase activity in the CNS may deliver attractive targets for drug development.

\section{Methods}

Sequencing of patient DNA. The intronic ATP6AP2 variant in the patient was identified by use of the XLID Next-Gen panel sequencing screen (Ambry Genetics) and verified by Sanger sequencing at Greenwood Genetic Center (Greenwood, South Carolina, USA). The Ambry XLID Next-Gen Panel targets detection of mutations in 81 genes by sequencing of all coding domains plus at least 10 bases into the $5^{\prime}$ and $3^{\prime}$ ends of all introns.

Generation and characterization of iPSCs. Fibroblasts from the ATP6AP2 (c.301-11_301-10delTT) patient were reprogrammed into the induced pluripotent stem cell (iPSC) line PB41, using a nonintegrating CytoTune Sendai viral vector kit (Life Technologies, A13780) according to the manufacturer's instructions with slight modifications. Briefly, reprogramming was achieved by overnight transduction of $1 \times 10^{5}$ fibroblasts in low-serum-containing fibroblast medium (FibroGRO-LS, Millipore) at a multiplicity of infection of 6 
A

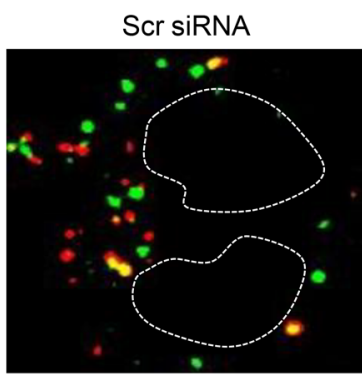

ATP6AP2 SIRNA + fl-ATP6AP2

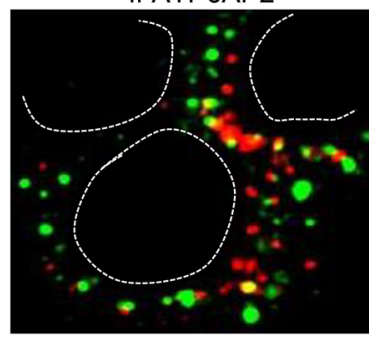

ATP6AP2 siRNA + empty vector

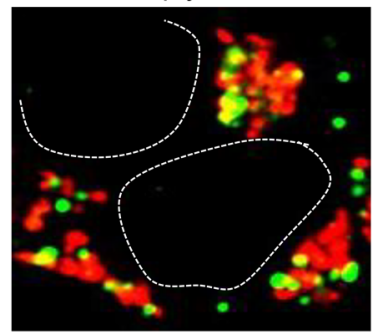

ATP6AP2 SiRNA + ATP6AP2 2 e4

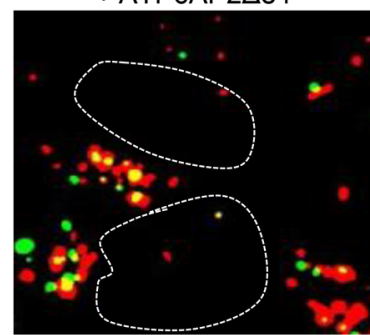

B

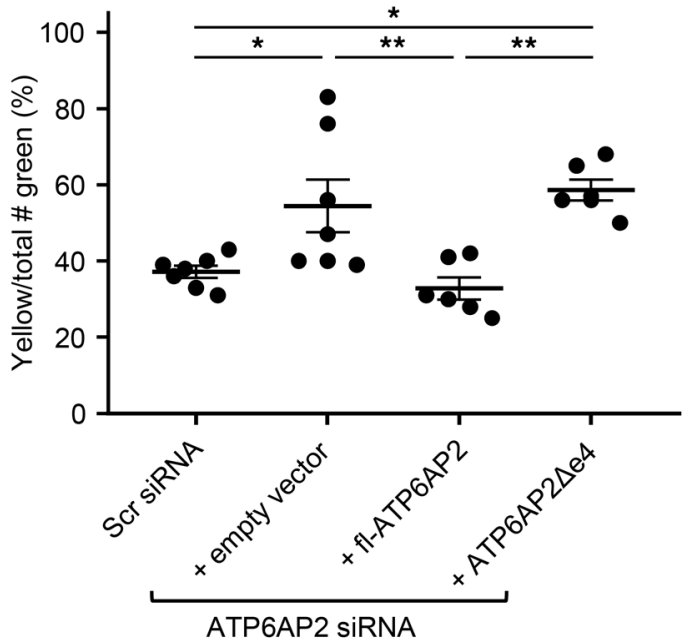

C

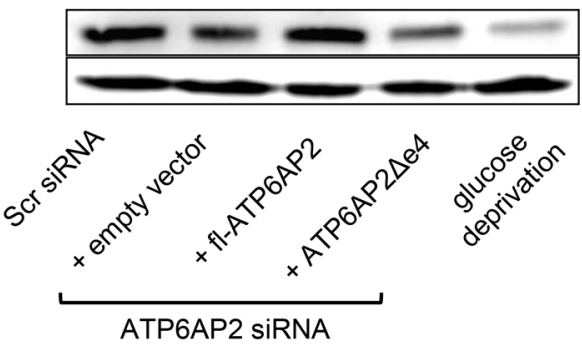

V1B2

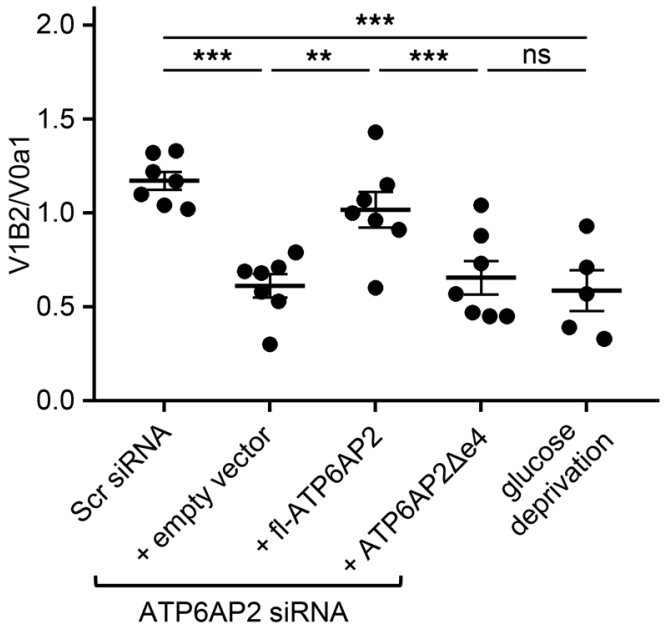

Figure 9. Enhanced vesicular fusion and reduction of membrane-associated V-ATPase holoenzyme in HeLa cells following ATP6AP2 knockdown. (A) Endosome-lysosome fusion studied by time-lapse microscopy. Representative images of increased fusion and perinuclear clustering following ATP6AP2 knockdown are obtained from merged single frames of Supplemental Videos 1-4. Dashed lines: nucleus. (B) The number of yellow vesicles resulting from the fusion of lysosomes labeled with LysoTracker (Red) and endosomes labeled with Oregon green 488. Data show individual values and mean \pm SEM; $n=$ 6-7 from 3 independent experiments. ${ }^{*} P<0.05,{ }^{* *} P<0.01$, 1-way ANOVA followed by Bonferroni's post test. (C) Membrane-bound V1B2 subunit of the V1 sector and VOa1 subunit of the VO sector of the V-ATPase of transfected HeLa cells were analyzed by Western blot (left). The band intensity was quantified (right) as a ratio of V1B2 to V0a1. Data show individual values and mean $\pm \mathrm{SEM} ; n=5-7$ from 3 independent experiments. ${ }^{* *} P<0.01,{ }^{* * *} P<0.001 ;$ NS, $P>0.05$; 1-way ANOVA followed by Bonferroni's post test.

(MOI6). Forty-eight hours later, transduced cells were passaged onto a freshly ES-qualified Matrigel-coated (BD Biosciences) 60-mm dish in FibroGRO-LS medium. The next day, medium was switched to Nutristem medium (Miltenyi Biotec) and changed every day until the emergence of reprogrammed colonies. iPSC colonies were identified under a stereomicroscope (Lynx, Vision Engineering) at day 25 after transduction and were manually picked and plated onto Mitomycin C-inactivated (Sigma-Aldrich) mouse embryonic fibroblasts in KOSR medium composed of DMEM/F12 culture medium, supplemented with $20 \%$ KnockOut Serum Replacement, $0.1 \mathrm{mmol} / \mathrm{l}$ nonessential amino acids, $1 \mathrm{mmol} / \mathrm{l}$ L-glutamine, $0.1 \mathrm{mmol} / \mathrm{l}$ 2-mercaptoethanol, penicillin/streptomycin (all from Life Technologies), and $12.5 \mathrm{ng} / \mathrm{ml}$ recombinant human basic FGF (Miltenyi Biotec). Control iPSCs were obtained from reprogramming of human foreskin fibroblasts (FibroGRO Xeno-Free Human Foreskin Fibroblasts, Millipore) with a Stemgent mRNA Reprogramming kit (Miltenyi Biotec) according to the manufacturer's instructions. The iPSC colonies were further expanded in KOSR medium onto inactivated mouse embryonic fibroblasts. All the cultures were performed at $37^{\circ} \mathrm{C}$ in a $5 \% \mathrm{CO}_{2}$ atmosphere. The iPSC lines were characterized by FACS analysis for the expression of pluripotent stem cell surface markers. Briefly, $10^{5}$ cells were stained with a combination of BD Horizon V450-conjugated mouse anti- 
human SSEA4 (Clone MC813-70, BD Biosciences), FITC-conjugated mouse anti-human HESCA1 (clone 051007-4A5, Millipore), phosphatidylethanolamine-conjugated (PE-conjugated) rat anti-mouse SSEA3 (clone MC631, BD Biosciences), and Alexa Fluor 647-conjugated mouse anti-human TRA-1-60 (clone TRA-1-60, BD Biosciences) according to the manufacturer's recommendations. Cells were analyzed on a MACSQuant flow cytometer (Miltenyi Biotec) using MACSQuantify software. The genomic integrity was assessed by karyotyping according to standard procedures. Following reprogramming, fl-ATP6AP2 and ATP6AP2 $\triangle \mathrm{e} 4$ transcripts were present. Pluripotency of the iPSC line was assessed by teratoma formation assays. Six-week-old NSG mice were subjected to intramuscular injection of $2 \times 10^{6}$ to $3 \times 10^{6}$ iPSCs. After 8 weeks, teratomas were dissected and fixed in $4 \%$ paraformaldehyde (PFA) and samples embedded in paraffin and stained with $\mathrm{H} \& \mathrm{E}$.

For re-expression of fl-ATP6AP2, the patient iPSC line was transduced with a pHAGE lentiviral vector (60) carrying mCherry cDNA and the cDNA of the fl-ATP6AP2. The patient control line was generated with the same vector without the ATP6AP2 cDNA. The mCherry-expressing cells were isolated by FACS.

Neuronal differentiation of iPSCs. Differentiation of iPSCs into cortical neurons was performed as described previously (61). Briefly, iPSC colonies were detached by treatment with type IV collagenase and kept in suspension in low-attachment plates (Corning) as embryoid bodies (EBs) during 5 days in iPSC culture media without FGF2 containing $10 \mu \mathrm{M}$ SB421542 (Sigma-Aldrich) and $20 \mu \mathrm{M}$ dorsomorphin (Sigma-Aldrich). EBs were then plated in polyornithine/ laminin-coated dishes and kept in culture for 7 extra days in neural precursor media (NPC: Neurobasal, B27 minus Vitamin A [Gibco], FGF2 [20 ng/ $\mu \mathrm{l}]$, and EGF $[20 \mathrm{ng} / \mu \mathrm{l}])$ until neural rosettes were observed. These rosettes were then picked manually and dissociated into single-cell suspension using trypsin (TrypLETM, Gibco) and plated at a density of $1.5 \times 10^{5}$ cells $/ \mathrm{mm}^{2}$ in neuronal differentiation media (NDM media: Neurobasal, B27 minus Vitamin A, BDNF [10 $\mathrm{ng} / \mu \mathrm{l}]$, GDNF [10 ng/ $\mu \mathrm{l}]$, and cAMP [100 $\mu \mathrm{M}])$. Western blots for p62 and LC3B were performed according to guidelines for autophagy assays (62).

Calcium imaging. The iPSC-derived neurons at 45 DIV were bulk-loaded with $5 \mu \mathrm{M}$ of Fluo4-AM (Life Technologies) in culture medium for 15 minutes at $37^{\circ} \mathrm{C}$. Then the culture medium was removed and replaced by an imaging medium: MEM, $4 \mathrm{mM}$ sodium bicarbonate, $20 \mathrm{mM}$ HEPES, $2 \mathrm{mM}$ GlutaMAX, $33 \mathrm{mM}$ D-glucose, $\mathrm{B} 27$, and N-2 serum. During imaging, iPSCs were perfused at a rate of $1 \mathrm{ml} / \mathrm{min}$ with imaging medium at $32^{\circ} \mathrm{C}$. Images were obtained with an upright microscope DM6000CFS (Leica Microsystems) equipped with a $\times 250.95$ numerical aperture water-immersion objective and a CCD camera (DFC 360 FX, Leica Microsystems). Illumination was by a short-arc HXP lamp (Osram); exposure times were 0.1-0.2 seconds. Excitation and dichroic filters were D480/40 nm and $505 \mathrm{~nm}$, respectively. Signals were acquired behind an emission filter (527/30 $\mathrm{nm}$ ). Recorded fluorescence movies ( 1 image per second during 5 minutes) were first corrected for movement artifacts using the StackReg plug-in for Image (NIH). The calcium signal for each detected cell was extracted from stable portions of movies. In these traces, the large deflections indicate calcium events that correspond to the increase of neuronal activity. The onset of each deflection was marked and used for building Rasterplots (MATLAB).
Fibroblast cell culture. Explants of 3-mm dermal biopsies were minced and placed in a $60-\mathrm{mm}$ tissue culture dish under a sterile coverslip held down by sterilized silicon grease. Fibroblast medium (DMEM supplemented with 10\% FCS, GlutaMAX, and penicillin/ streptomycin [Invitrogen]) was added, and dishes were incubated at $37^{\circ} \mathrm{C}$ in a humidified $5 \% \mathrm{CO}_{2}$ atmosphere with media exchange every 5 days. Fibroblast outgrowths were harvested by trypsinization, expanded in a T25 flask in fibroblast medium, and allowed to reach approximately $90 \%$ confluence before freezing or splitting for reprogramming as described below. For reprogramming, fibroblasts were used within the first 3 passages from biopsy or within 1 passage after a thawing.

Electron microscopy. Cells were fixed with $4 \%$ PFA and $2.5 \%$ glutaraldehyde in $0.1 \mathrm{M}$ phosphate buffer (1 hour) and postfixed in $\mathrm{OsO}_{4}$ (40 minutes) at $4^{\circ} \mathrm{C}$. Samples were dehydrated in an ascending series of ethanol, and embedded in epoxy resin. Semithin sections $(0.5 \mu \mathrm{m})$ were stained with toluidine blue and viewed with a Provis Olympus microscope. Images were acquired with a Coolsnap CCD camera. Ultrathin sections (40 nm thick) were mounted in 200 meshes, cut, and double-stained with uranyl acetate and lead citrate before observation with a Philips (CM-100) electron microscope. Digital images were obtained with a CCD camera (Gatan Orius). Morphometric analysis was performed with the software Digital Micrograph on 2 different experiments of each genotype. For lysosomal diameter, lysosomes were identified by their morphology as described previously (63) and their diameter measured in 10 neurons per genotype.

Animals. Mouse strains were on a C57BL/6 genetic background and have been described earlier: Atp6ap2 flox (10), Emx1-Cre (64), and CamK2a-Cre $(65,66)$. All procedures were designed to minimize animal suffering and were carried out in accordance with European Economic Community guidelines (2010/63/UE) for the care and use of experimental animals and French National Committee guidelines $(87 / 848)$ for the use of laboratory animals.

Behavior tests. Open field test and contextual fear conditioning were performed as described previously (67). The open field test was conducted in a brightly illuminated square arena. Mice were placed in the center of the arena and allowed to explore the area for 30 minutes. Behavior was tracked using a ceiling-mounted camera (Panasonic WV BP332). For contextual fear conditioning, mice were placed in a conditioning chamber and allowed to habituate for 3 minutes, followed by 3 consecutive foot shocks $(1.0 \mathrm{~mA}, 50 \mathrm{~Hz}, 1$ second) separated by 1 minute. Animals remained in the chamber for 1 minute after the delivery of the last foot shock. Testing occurred 24 hours after in the same chamber, and freezing was scored for a total of 5 minutes. Sessions were recorded and freezing was manually scored blinded for genotype. Freezing was defined as complete absence of movement, except for respiration. Data were expressed as percentage of time freezing.

Immunohistochemistry. Sections of $40 \mu \mathrm{m}$ (P15, 6 months) and 60 $\mu \mathrm{m}$ (E12, E13, E14) were prepared using a VT1000S vibratome (Leica Biosystems) and maintained at $-20^{\circ} \mathrm{C}$ in cryoprotectant tissue collecting buffer (30\% ethylene glycol, 30\% glycerol in $0.024 \mathrm{M}$ phosphate buffer). For frozen sections, brains were cryostat-sectioned into 5- to $10-\mu \mathrm{m}$ slices (Leica CM1800, Leica Biosystems). Nonspecific binding was blocked by pretreatment with $5 \%$ normal goat serum in PBS containing $0.2 \%$ gelatin. The sections were incubated in primary antibodies overnight at $4^{\circ} \mathrm{C}$ in PBS $0.2 \%$ gelatin or $0.4 \%$ saponin. The list of antibodies is provided in Supplemental Table 1. BrdU and Ki67 staining 
was performed as described previously (53). Alexa Fluor 488 or Alexa Fluor 555 fluorescent secondary antibodies (Invitrogen) were used for Leica laser-scanning 2-photon microscopy (Leica TCS SP5, Leica Biosystems), and secondary antibodies (Jackson ImmunoResearch) were conjugated to Atto 565 (ATTO-TEC) and DY 485 (Dyomics) and used for 2-color STED microscopy on a custom-built setup (68).

Cell culture and transfection. HeLa (CCL-2) and HEK293T (CRL3216) cells were obtained from the American Type Culture Collection and cultured in DMEM (Invitrogen) containing 10\% FCS. Constructs, scramble, and siRNAs targeting the 3'-UTR (Dharmacon) were transfected into HeLa cells using Lipofectamine 2000 (Life Technologies) according to the manufacturer's instructions.

Vesicular pH measurement, fluorescent microscopy, and image processing. Vesicular $\mathrm{pH}$ was determined as described previously (69). Briefly, HeLa cells were seeded in 8-well Labtek II chambers (Thermo Fisher Scientific) and transfected with siRNA with or without rescue constructs. Twenty-four hours later, cells were incubated in $1 \mathrm{mg} / \mathrm{ml}$ LysoSensor Yellow/Blue dextran (Invitrogen) for 1 hour, with or without $100 \mathrm{nM}$ bafilomycin. Cells were then washed twice in PBS. For each experiment, a $\mathrm{pH}$ standard curve was obtained by treatment of the cells with clamp buffers at $\mathrm{pH}$ 3.5-7.5. Cells were equilibrated in clamp buffer $\mathrm{pH}$ 3.5-7.5 $\left(5 \mathrm{mM} \mathrm{NaCl}, 115 \mathrm{mM} \mathrm{KCl}, 1.2 \mathrm{mM} \mathrm{MgSO}_{4}, 25 \mathrm{mM}\right.$ 2-morpholinoethanesulfonic acid [MES], $\mathrm{pH}$ 3.5-7.5) followed by washing in clamp buffer supplemented with $25 \mu \mathrm{M}$ monensin and $10 \mu \mathrm{M}$ nigericin (Sigma-Aldrich) for 20 minutes before imaging. All equilibration steps were performed in a $37^{\circ} \mathrm{C}, 5 \% \mathrm{CO}_{2}$ incubator. Imaging was performed on a heated stage $\left(37^{\circ} \mathrm{C}\right)$. Cells were excited with attenuated UV light $(357-373 \mathrm{~nm})$ and observed in blue (W1; 417-483 nm) and yellow (W2; 490-530 nm) regions of the spectra. Images were collected on a Leica DM IRBE, using a $\times 63 / 1.25$ NA objective over a period of 5 minutes. Sixteen-bit images were processed to subtract background fluorescence, and then ratio (W1/W2) images were generated using the image calculator function in ImageJ. Regions of interest corresponding to vesicles were generated using $\mathrm{W} 2$ and applied to ratio images to determine the $\mathrm{W} 1 / \mathrm{W} 2$ ratio value of the vesicles for each $\mathrm{pH}$ clamp buffer. A standard curve was generated in GraphPad Prism to assign a predicted $\mathrm{pH}$ for W1/W2 values.

$V$-ATPase-dependent acidification of lysosomes. V-ATPase activity on lysosomes was investigated as described previously (70). Briefly, HEK293T cells were plated into $10-\mathrm{cm}$ plates coated with poly-Dlysine (Millipore) and transfected with siRNA and rescue constructs. The next day, the medium was replaced to $10 \%$ FCS/DMEM containing $2.0 \mathrm{mg} / \mathrm{ml}$ FITC-dextran (Sigma-Aldrich), and cells were incubated overnight. After starvation for 6 hours in serum-free DMEM, cells were scraped into fraction buffer and lysed by passage of the cell suspension through a 27-gauge needle 8 times. After removal of intact cells and nuclei by centrifugation at $2000 \mathrm{~g}$ for 10 minutes, the supernatants were centrifuged at $16,100 \mathrm{~g}$ for 15 minutes. Then the pellets, including FITC-dextran-containing lysosomes, were resuspended in $100 \mu \mathrm{l}$ fraction buffer, and concanamycin A-sensitive V-ATPase proton pumping was assessed by a spectrophotometer (Varioskan LUX Multimode Microplate Reader, Thermo Fisher Scientific).

EGFR degradation. Epidermal growth factor receptor (EGFR) degradation was studied as described previously (71) using a HeLa cell line with stable knockdown of ATP6AP2. Briefly, control (Control HeLa SilenciX, catalog 00301-00001, Tebu-bio) and ATP6AP2-KD HeLa (ATP6AP2 HeLa SilenciX, catalog 00301-00042, Tebu-bio) cells were cultured in 6-well plates at $90 \%$ confluence with 10\% FCS/DMEM containing $125 \mathrm{mg} / \mathrm{ml}$ Hygromycin B (Invitrogen). Cells were starved with $0.1 \%$ FCS/DMEM (without antibiotics) for 20 hours and then preincubated with $10 \mathrm{mg} / \mathrm{ml}$ cycloheximide for 30 minutes and stimulated with $100 \mathrm{ng} / \mathrm{ml}$ recombinant human EGF (236-EG-200, R\&D Systems) for $0,30,60,120,180$, and 240 minutes, in triplicate. Cells were then washed with ice-cold PBS, lysed with lysis buffer (20 mM HEPES-KOH pH 7.4, 100 mM KCl, 0.5 mM EDTA, 10 mM NaF, 1\% Triton X-100, $1 \mathrm{mM} \mathrm{Na}_{3} \mathrm{VO}_{4}, 10 \mathrm{mM} \mathrm{Na} \mathrm{P}_{2} \mathrm{O}_{7}, 0.1 \mathrm{mM} \mathrm{Na}_{2} \mathrm{MoO}_{4}, \beta$-glycerolphosphate, protease inhibitor cocktail [Complete, Roche Diagnostics]), and scraped. Cell lysates were analyzed by 7.5\% SDS-PAGE and Western blotting with anti-EGFR (sc-03, Santa Cruz Biotechnology).

Cloning and expression. Expression vectors for either human fl-ATP6AP2 cDNA or ATP6AP2 $\triangle$ e 4 were generated from the patient with ATP6AP2 variant c.321C>T (p.D107D) (OMIM 300423) (72) and ligated into the KpnI/EcoRI site in pcDNA3.1(+) vector (Invitrogen) (Supplemental Table 2).

Vesicle fusion and live cell imaging. Endosome-lysosome fusion assays were adapted from Bright et al. (73). Briefly, HeLa cells were seeded in 8-well Labtek II chambers and transfected with siRNA with or without rescue construct. Then cells were loaded with 50 nM LysoTracker Red (Life Technologies) for 4 hours followed by incubation in conjugate-free medium for 20 hours. Cells were then loaded with dextran Oregon green 488 (10,000 MW, anionic, fixable; Life Technologies) for 10 minutes followed by a 5-minute chase in conjugate-free $\mathrm{CO}_{2}$-independent medium (Invitrogen). Cells were washed 3 times with $\mathrm{PBS}$ and transferred to the heated stage, and spinning-disk images were acquired using a $\times 63 / 0.15$ NA objective on a Nikon Eclipse Ti microscope, equipped with an Evolve 512/EM-CCD camera (Photometrics) and a CSUX1-A1 (Yokogawa) confocal scanner. Fluorescence was excited with a 491-nm laser and detected with a 525/39 nm filter for Oregon green 488. A 561-nm laser and 605/64 nm filter were used for LysoTracker Red. MetaMorph software (Molecular Devices) was used to collect the data and ImageJ to assemble the movies.

Membrane fractionation and Western blotting. To prepare membrane fractions, HeLa cells were homogenized with a Teflon potter in sucrose buffer, and the postnuclear supernatant was fractionated into cytosolic and membrane fractions by ultracentrifugation (60 minutes, $100,000 \mathrm{~g}$ ) as described previously (74). Alternatively, cell lysate was obtained by treatment of cells with lysis buffer (Cell Signaling) containing protease and phosphatase inhibitors. Total protein was separated on $10 \%$ or $16 \%$ SDS-PAGE and transferred to PVDF membranes (Millipore). The membranes were blocked with 5\% nonfat dry milk or $5 \%$ BSA in $20 \mathrm{mM}$ Tris- $\mathrm{HCl} \mathrm{pH} \mathrm{7.4,} 150 \mathrm{mM} \mathrm{NaCl}$ with Triton $\mathrm{X}-100$ for 30 minutes at room temperature and incubated overnight with primary antibodies at $4^{\circ} \mathrm{C}$. Membranes were incubated with alkaline phosphatase-conjugated secondary antibodies (1:5000; Jackson ImmunoResearch) for 1 hour at room temperature. AttoPhos AP Fluorescent Substrate System (Promega) was used for detection, and quantification of bands was performed using ImageJ software. The primary antibodies used are listed in Supplemental Table 1.

Statistics. R (version 2.14.0) (http://www.R-project.org) and GraphPad Prism software (version 8.0.1) were used for statistical analyses. A $P$ value less than 0.05 was considered significant. Mann-Whitney $U$ test, Student's $t$ test (unpaired, 2-tailed), Kruskal-Wallis test, 1-way ANOVA, or 2-way repeated-measures ANOVA was performed for statistical analysis based on group numbers and data distributions. Bonferroni's correction for parametric analy- 
sis or Dunn's multiple comparison for nonparametric analysis was conducted for multiple post hoc comparisons. Data normality was verified by Shapiro-Wilk test before performance of parametric or nonparametric tests (75).

Study approval. Informed consent was obtained from the parents of the patient carrying the ATP6AP2 (c.321C $>$ T) variant, and the study was approved by the Institutional Review Committee (IRB\#26) of Self Regional Healthcare, Greenwood, South Carolina, USA. Informed consent was obtained from the parents of the patient carrying the ATP6AP2 (c.301-11_301-10delTT) variant, and the study was approved by the Research Ethics Board of Mount Sinai Hospital, Toronto, Ontario, Canada. The parents gave informed consent for blood sampling and skin biopsy to generate iPSC lines. The animal study protocol was approved by the Institutional Animal Care Committee of College de France, Paris, France, license number 01513.01.

\section{Author contributions}

M Groszer and GN conceptualized the study. TH, ACS, TL, OF, CCD, NG, CM, LS, JAB, HY, MAL, M. Guillon, KK, JWN, JT, MR, SI, VE, KT, GN, and M. Groszer conducted experiments. TH, ACS, TL, OF, CCD, NG, CM, TG, JAB, HY, MAL, M. Guillon, KK, JWN, JT, SI, PC, VE, ABG, KT, GN, and M. Groszer analyzed experimental data. DC, JWN, KS, SB, RML, MGM, KRH, and CES acquired and analyzed clinical data. JH provided reagents. TH, ACS, DC, TL, GN, and M. Groszer wrote the manuscript.

\section{Acknowledgments}

This work was supported in part by grants from Agence Nationale de la Recherche (ANR) (to M Groszer); Ecole des Neurosciences de Paris (to $M$ Groszer and VE); INSERM/CNRS ATIP-AVENIR programme (to M Groszer); Fondation pour la Recherche Médicale en France (to M Groszer); l'Association "Schizo oui" (to M Groszer); Institut de France-Fondation NRJ (to M Groszer); DIM Biothérapies-Stem-Pôle (to M Groszer); Investissements d'Avenir/program (Labex Biopsy) managed by the ANR under reference ANR-11-IDEX-0004-02 (to M Groszer); a France-Stanford grant (to M Groszer); Association Vaincre les Maladies Lysosomales (to M Groszer); Fondation Lejeune (to M Groszer); Naturalia and Biologia and La Fondation du Rein (to GN); Company of Biologists (to ACS); a Grant-in-Aid for Scientific Research (23790242 and 25860156) and postdoctoral fellowship from the Japan Society for the Promotion of Science (to TH); grants ANR Investissement Avenir Infrastructure INGESTEM (to ABG); Japan Science and Technology Agency, CREST program (to KT and HY); Paris Des- cartes University, Collaborative Project 1199PJ05 (to MAL, M Guillon, and VE); Center for Competences in Nano-science (C'Nano) and the People Programme (Marie Curie Actions) of the European Union's Seventh Framework Programme (FP7) under REA grant agreement PIEF-GA-2011-297917 (to MAL); the NIH (R01HD026202, R01NS073854); and the South Carolina Department of Disabilities and Special Needs (to CES). We thank Ricardo Dolmetsch (Stanford University, Palo Alto, California, USA) for support to establish neuronal differentiation of human iPSCs; Carsten A. Wagner (University of Zurich, Zurich, Switzerland) for the anti-ATP6V1B2 antibody; Anny Anglo, Patrick Le Griel, and Isabelle Genois for electron microscopy imaging; Christophe Tourain for custom-built electronics; Patrice Jegouzo for support with mechanical parts; Ariel de Nardo and Alain Prochiantz (Collège de France, Paris, France) for access to microscopes; and Chika Takahashi and Takefumi Mori (Tohoku Medical and Pharmaceutical University, Sendai, Japan) for assistance with cell culture experiments. Dedicated to the memory of Ethan Francis Schwartz.

Address correspondence to: Matthias Groszer, Institut du Fer à Moulin, Inserm, 17 rue du Fer à Moulin, 75005 Paris, France. Phone: 33.0.1.45.87.61.48; Email: matthias.groszer@inserm.fr. Or to: Genevieve Nguyen, Centre for Interdisciplinary Research in Biology (CIRB), UMR INSERM U1050/CNRS 7241, Collège de France, 11 place Marcelin Berthelot, 75231 Paris Cedex 05, France. Phone: 33.0.1.44.27.16.89; Email: genevieve-1.nguyen@ diplomatie.gouv.fr.

TH's present address is: Division of Nephrology and Endocrinology, Tohoku Medical and Pharmaceutical University, Miyagino, Sendai, Japan; and Division of Nephrology, Endocrinology and Vascular Medicine, Department of Medicine, Tohoku University Graduate School of Medicine, Aoba, Sendai, Japan.

ACS's present address is: Janssen Research \& Development, Janssen Pharmaceutica (Johnson \& Johnson), Dr. Paul Janssen Research Center, Beerse, Belgium.

KK's present address is: Department of Molecular Biochemistry, Iwate Medical University School of Medicine, Iwate, Japan.

MAL's present address is: Center for Integrative Physiology and Molecular Medicine (CIPMM), Medical Faculty, Saarland University, Homburg (Saar), Germany.
1. Forgac M. Vacuolar ATPases: rotary proton pumps in physiology and pathophysiology. Nat Rev Mol Cell Biol. 2007;8(11):917-929.

2. Wang D, Hiesinger PR. The vesicular ATPase: a missing link between acidification and exocytosis. J Cell Biol. 2013;203(2):171-173.

3. Laplante M, Sabatini DM. Regulation of mTORC1 and its impact on gene expression at a glance. J Cell Sci. 2013;126(pt 8):1713-1719.

4. Nixon RA. The role of autophagy in neurodegenerative disease. Nat Med.2013;19(8):983-997.

5. Nguyen G, Delarue F, Burcklé C, Bouzhir L,
Giller T, Sraer JD. Pivotal role of the renin/ prorenin receptor in angiotensin II production and cellular responses to renin. JClin Invest. 2002;109(11):1417-1427.

6. Cousin C, Bracquart D, Contrepas A, Corvol P, Muller L, Nguyen G. Soluble form of the (pro) renin receptor generated by intracellular cleavage by furin is secreted in plasma. Hypertension. 2009;53(6):1077-1082.

7. Bader M. The second life of the (pro)renin receptor. J Renin Angiotensin Aldosterone Syst. 2007;8(4):205-208.
8. Cruciat CM, et al. Requirement of prorenin receptor and vacuolar $\mathrm{H}^{+}$-ATPase-mediated acidification for Wnt signaling. Science. 2010;327(5964):459-463.

9. Kinouchi K, et al. The (pro)renin receptor/ ATP6AP2 is essential for vacuolar $\mathrm{H}^{+}$-ATPase assembly in murine cardiomyocytes. Circ Res. 2010;107(1):30-34.

10. Riediger F, et al. Prorenin receptor is essential for podocyte autophagy and survival. J Am Soc Nephrol.2011;22(12):2193-2202.

11. Guida MC, et al. ATP6AP2 functions as a V-AT- 
Pase assembly factor in the endoplasmic reticulum. Mol Biol Cell. 2018;29(18):2156-2164.

12. Ramser J, et al. A unique exonic splice enhancer mutation in a family with $\mathrm{X}$-linked mental retardation and epilepsy points to a novel role of the renin receptor. Hum Mol Genet. 2005;14(8):1019-1027.

13. Korvatska O, et al. Altered splicing of ATP6AP2 causes $\mathrm{X}$-linked parkinsonism with spasticity (XPDS). Hum Mol Genet. 2013;22(16):3259-3268.

14. Piton A, Redin C, Mandel JL. XLID-causing mutations and associated genes challenged in light of data from large-scale human exome sequencing. Am J Hum Genet. 2013;93(2):368-383.

15. Rujano MA, et al. Mutations in the X-linked ATP6AP2 cause a glycosylation disorder with autophagic defects. J Exp Med. 2017;214(12):3707-3729.

16. Gupta HV, Vengoechea J, Sahaya K, Virmani T. A splice site mutation in ATP6AP2 causes $\mathrm{X}$-linked intellectual disability, epilepsy, and parkinsonism. Parkinsonism Relat Disord. 2015;21(12):1473-1475.

17. Li W, et al. Neuron-specific (pro)renin receptor knockout prevents the development of salt-sensitive hypertension. Hypertension. 2014;63(2):316-323.

18. Hedera P, Alvarado D, Beydoun A, Fink JK. Novel mental retardation-epilepsy syndrome linked to Xp21.1-p11.4. Ann Neurol. 2002;51(1):45-50.

19. Dubos A, et al. Conditional depletion of intellectual disability and Parkinsonism candidate gene ATP6AP2 in fly and mouse induces cognitive impairment and neurodegeneration. Hum Mol Genet. 2015;24(23):6736-6755.

20. Desmet FO, Hamroun D, Lalande M, Collod-Béroud G, Claustres M, Béroud C. Human Splicing Finder: an online bioinformatics tool to predict splicing signals. Nucleic Acids Res. 2009;37(9):e67.

21. Götz M, Huttner WB. The cell biology of neurogenesis. Nat Rev Mol Cell Biol. 2005;6(10):777-788.

22. Kanda A, et al. Atp6ap2/(pro)renin receptor interacts with Par3 as a cell polarity determinant required for laminar formation during retinal development in mice. J Neurosci. 2013;33(49):19341-19351.

23. Sansom SN, et al. The level of the transcription factor Pax6 is essential for controlling the balance between neural stem cell self-renewal and neurogenesis. PLoS Genet. 2009;5(6):e1000511.

24. Kim S, et al. The apical complex couples cell fate and cell survival to cerebral cortical development. Neuron. 2010;66(1):69-84.

25. Oshima $Y$, et al. Prorenin receptor is essential for normal podocyte structure and function. J Am Soc Nephrol. 2011;22(12):2203-2212.

26. Settembre C, Fraldi A, Medina DL, Ballabio A. Signals from the lysosome: a control centre for cellular clearance and energy metabolism. Nat Rev Mol Cell Biol. 2013;14(5):283-296.

27. Korolchuk VI, et al. Lysosomal positioning coordinates cellular nutrient responses. Nat Cell Biol. 2011;13(4):453-460.

28. Lalonde R, Strazielle C. Brain regions and genes affecting limb-clasping responses. Brain Res Rev. 2011;67(1-2):252-259.

29. Langford-Smith A, et al. Hyperactive behaviour in the mouse model of mucopolysaccharidosis
IIIB in the open field and home cage environments. Genes Brain Behav. 2011;10(6):673-682.

30. Anagnostaras SG, Gale GD, Fanselow MS. Hippocampus and contextual fear conditioning: recent controversies and advances. Hippocampus. 2001;11(1):8-17.

31. Gaspard N, et al. An intrinsic mechanism of corticogenesis from embryonic stem cells. Nature. 2008;455(7211):351-357.

32. Eiraku M, et al. Self-organized formation of polarized cortical tissues from ESCs and its active manipulation by extrinsic signals. Cell Stem Cell. 2008;3(5):519-532.

33. Wolfe DM, Lee JH, Kumar A, Lee S, Orenstein SJ, Nixon RA. Autophagy failure in Alzheimer's disease and the role of defective lysosomal acidification. Eur J Neurosci. 2013;37(12):1949-1961.

34. Heck N, Golbs A, Riedemann T, Sun JJ, Lessmann V, Luhmann HJ. Activity-dependent regulation of neuronal apoptosis in neonatal mouse cerebral cortex. Cereb Cortex. 2008;18(6):1335-1349.

35. Illes S, et al. Intrinsically active and pacemaker neurons in pluripotent stem cell-derived neuronal populations. Stem Cell Reports. 2014;2(3):323-336.

36. Mattson MP. Calcium and neurodegeneration. Aging Cell. 2007;6(3):337-350.

37. Kinouchi $\mathrm{K}$, et al. The role of individual domains and the significance of shedding of ATP6AP2/ (pro)renin receptor in vacuolar $\mathrm{H}(+)$-ATPase biogenesis. PLoS One. 2013;8(11):e78603.

38. Lin $\mathrm{Q}$, Yang $\mathrm{W}$, Cerione RA. Measurement of epidermal growth factor receptor turnover and effects of Cdc42. Meth Enzymol. 2006;406:614-625.

39. Klionsky DJ, Elazar Z, Seglen PO, Rubinsztein DC. Does bafilomycin A1 block the fusion of autophagosomes with lysosomes? Autophagy. 2008;4(7):849-850.

40. Coonrod EM, et al. Homotypic vacuole fusion in yeast requires organelle acidification and not the V-ATPase membrane domain. Dev Cell. 2013;27(4):462-468.

41. Di Giovanni J, et al. V-ATPase membrane sector associates with synaptobrevin to modulate neurotransmitter release. Neuron. 2010;67(2):268-279.

42. Hiesinger PR, et al. The v-ATPase VO subunit a1 is required for a late step in synaptic vesicle exocytosis in Drosophila. Cell. 2005;121(4):607-620.

43. Lee SH, et al. v-ATPase VO subunit d2-deficient mice exhibit impaired osteoclast fusion and increased bone formation. Nat Med. 2006;12(12):1403-1409.

44. Peri F, Nüsslein-Volhard C. Live imaging of neuronal degradation by microglia reveals a role for v0-ATPase a1 in phagosomal fusion in vivo. Cell. 2008;133(5):916-927.

45. Peters C, Bayer MJ, Bühler S, Andersen JS, Mann M, Mayer A. Trans-complex formation by proteolipid channels in the terminal phase of membrane fusion. Nature. 2001;409(6820):581-588.

46. Strasser B, Iwaszkiewicz J, Michielin O, Mayer A. The V-ATPase proteolipid cylinder promotes the lipid-mixing stage of SNARE-dependent fusion of yeast vacuoles. EMBO J. 2011;30(20):4126-4141.

47. Sun-Wada GH, Toyomura T, Murata Y, Yamamoto A, Futai M, Wada Y. The a3 isoform of V-ATPase regulates insulin secretion from pancreatic beta-cells. J Cell Sci. 2006;119(pt 21):4531-4540. 48. Williamson WR, Wang D, Haberman AS,
Hiesinger PR. A dual function of VO-ATPase a1 provides an endolysosomal degradation mechanism in Drosophila melanogaster photoreceptors. J Cell Biol. 2010;189(5):885-899.

49. Williamson WR, Hiesinger PR. On the role of v-ATPase VOa1-dependent degradation in Alzheimer disease. Commun Integr Biol. 2010;3(6):604-607.

50. Bernasconi P, Rausch T, Struve I, Morgan L, Taiz L. An mRNA from human brain encodes an isoform of the B subunit of the vacuolar H(+)-ATPase. J Biol Chem. 1990;265(29):17428-17431.

51. Poorkaj P, et al. A novel X-linked four-repeat tauopathy with Parkinsonism and spasticity. Mov Disord.2010;25(10):1409-1417.

52. Lange C, Prenninger S, Knuckles P, Taylor V, Levin $\mathrm{M}$, Calegari F. The $\mathrm{H}(+)$ vacuolar ATPase maintains neural stem cells in the developing mouse cortex. Stem Cells Dev. 2011;20(5):843-850.

53. Chenn A, Walsh CA. Regulation of cerebral cortical size by control of cell cycle exit in neural precursors. Science. 2002;297(5580):365-369.

54. Nixon RA, Yang DS, Lee JH. Neurodegenerative lysosomal disorders: a continuum from development to late age. Autophagy. 2008;4(5):590-599.

55. Kole AJ, Annis RP, Deshmukh M. Mature neurons: equipped for survival. Cell Death Dis. 2013;4:e689.

56. Ludwig J, et al. Identification and characterization of a novel 9.2-kDa membrane sector-associated protein of vacuolar proton-ATPase from chromaffin granules. J Biol Chem. 1998;273(18):10939-10947.

57. Sihn G, Rousselle A, Vilianovitch L, Burckle C, Bader M. Physiology of the (pro)renin receptor: Wnt of change? Kidney Int. 2010;78(3):246-256.

58. Honasoge A, Sontheimer H. Involvement of tumor acidification in brain cancer pathophysiology. Front Physiol. 2013;4:316.

59. Harris H, Rubinsztein DC. Control of autophagy as a therapy for neurodegenerative disease. Nat Rev Neurol. 2011;8(2):108-117.

60. Thomas D, Mostoslavsky G. Efficient transduction of hematopoietic stem cells and its potential for gene correction of hematopoietic diseases. Methods Mol Biol. 2014;1114:441-450.

61. Paşca SP, et al. Using iPSC-derived neurons to uncover cellular phenotypes associated with Timothy syndrome. Nat Med.2011;17(12):1657-1662.

62. Barth S, Glick D, Macleod KF. Autophagy: assays and artifacts. J Pathol. 2010;221(2):117-124.

63. Wei J, et al. Enhanced lysosomal pathology caused by beta-synuclein mutants linked to dementia with Lewy bodies. J Biol Chem. 2007;282(39):28904-28914.

64. Gorski JA, Talley T, Qiu M, Puelles L, Rubenstein JL, Jones KR. Cortical excitatory neurons and glia, but not GABAergic neurons, are produced in the Emx1-expressing lineage. J Neurosci. 2002;22(15):6309-6314.

65. Fukaya M, Kato A, Lovett C, Tonegawa S, Watanabe M. Retention of NMDA receptor NR2 subunits in the lumen of endoplasmic reticulum in targeted NR1 knockout mice. Proc Natl Acad Sci US A. 2003;100(8):4855-4860.

66. Tsien JZ, et al. Subregion- and cell type-restricted gene knockout in mouse brain. Cell. 1996;87(7):1317-1326.

67. Kiyasova V, et al. A genetically defined morpho- 
logically and functionally unique subset of 5-HT neurons in the mouse raphe nuclei. J Neurosci. 2011;31(8):2756-2768.

68. Lauterbach MA, Guillon M, Soltani A, Emiliani V. STED microscope with spiral phase contrast. Sci Rep. 2013;3:2050.

69. Zhang X, et al. A role for presenilins in autophagy revisited: normal acidification of lysosomes in cells lacking PSEN1 and PSEN2. J Neurosci. 2012;32(25):8633-8648.
70. McGuire CM, Forgac M. Glucose starvation increases V-ATPase assembly and activity in mammalian cells through AMP kinase and phosphatidylinositide 3-kinase/Akt signaling. J Biol Chem. 2018;293(23):9113-9123.

71. Endo Y, et al. Regulation of clathrin-mediated endocytosis by p53. Genes Cells. 2008;13(4):375-386.

72. Contrepas A, et al. A role of the (pro)renin receptor in neuronal cell differentiation. Am JPhysiol Regul Integr Comp Physiol. 2009;297(2):R250-R257.
73. Bright NA, Gratian MJ, Luzio JP. Endocytic delivery to lysosomes mediated by concurrent fusion and kissing events in living cells. Curr Biol. 2005;15(4):360-365.

74. Trombetta ES, Ebersold M, Garrett W, Pypaert $\mathrm{M}$, Mellman I. Activation of lysosomal function during dendritic cell maturation. Science. 2003;299(5611):1400-1403.

75. Royston JP. Algorithm AS 181: the W-test for normality.J R Stat Soc Ser C Appl Stat. 1982;31:176-180. 